\title{
The Existence of Periodic Orbits and Invariant Tori for Some 3-Dimensional Quadratic Systems
}

\author{
Yanan Jiang, ${ }^{1}$ Maoan Han, ${ }^{1}$ and Dongmei Xiao ${ }^{2}$ \\ ${ }^{1}$ Department of Mathematics, Shanghai Normal University, Shanghai 200234, China \\ ${ }^{2}$ Department of Mathematics, Shanghai Jiaotong University, Shanghai 200240, China \\ Correspondence should be addressed to Maoan Han; mahan@shnu.edu.cn
}

Received 4 December 2013; Accepted 3 March 2014; Published 26 March 2014

Academic Editors: B. Hong and J. Li

Copyright (c) 2014 Yanan Jiang et al. This is an open access article distributed under the Creative Commons Attribution License, which permits unrestricted use, distribution, and reproduction in any medium, provided the original work is properly cited.

We use the normal form theory, averaging method, and integral manifold theorem to study the existence of limit cycles in LotkaVolterra systems and the existence of invariant tori in quadratic systems in $\mathbb{R}^{3}$.

\section{Introduction}

It is well known that $n$-dimensional generalized LotkaVolterra systems are widely used as the first approximation for a community of $n$ interacting species, each of which would exhibit logistic growth in the absence of other species in population dynamics. And this system is of wide interest in different branches of science, such as physics, chemistry, biology, evolutionary game theory, and economics. We refer the reader to the book of Hofbauer and Sigmund [1] for its applications. The existence of limit cycles and invariant tori for these models is interesting and significant in both mathematics and applications since the existence of stable limit cycles and invariant tori provided a satisfactory explanation for those species communities in which populations are observed to oscillate in a rather reproducible periodic manner (cf. [2-4] and references therein).

To study the bifurcation of Lotka-Volterra class, we consider three-dimensional generalized Lotka-Volterra systems

$$
\frac{d X_{i}(t)}{d t}=X_{i}(t)\left(\beta_{i}+\sum_{j=1}^{3} \alpha_{i j} X_{j}(t)\right), \quad i=1,2,3
$$

which describes the interaction of three species in a constant and homogeneous environment, where $X_{i}(t)$ is the number of individuals in the $i$ th population at time $t$ and $X_{i}(t) \geq 0$, $\beta_{i}$ is the intrinsic growth rate of the $i$ th population, the $\alpha_{i j}$ are interaction coefficients measuring the extent to which the $j$ th species affects the growth rate of the $i$ th, $\beta_{i}$ and $\alpha_{i j}$ are parameters, and the values of these parameters are not very small usually.

Over the last several decades, many researchers have devoted their effort to study the existence and number of isolated periodic solutions for system (1). There have been a series of achievements and unprecedented challenges on the theme even if system (1) is a competitive system (cf. [512]). In [13], Bobieński and Żołądek gave four components of center variety in the three-dimensional Lotka-Volterra class and studied the existence and number of isolated periodic solutions by certain Poincaré-Melnikov integrals of a new type. In [14], Llibre and Xiao used the averaging method to study the existence of limit cycles of three-dimensional Lotka-Volterra systems. In this paper, we will use the normal form theory to study the same question. And furthermore, we will give the existence of invariant tori in a system of the form (2).

This paper is organized as follows. In Section 2, we obtain some preliminary theorems about a normal form system of degree two in $\mathbb{R}^{3}$ with two small parameters $\lambda_{1}$ and $\lambda_{2}$ and other bounded parameters. In Section 3, we first change the system (1) into a system of the form

$$
\frac{d U}{d t}=u \varepsilon U+v V+\sum_{i+j+k=2} a_{i j k} U^{i} V^{j} W^{k}
$$




$$
\begin{gathered}
\frac{d V}{d t}=-v U+u \varepsilon V+\sum_{i+j+k=2} b_{i j k} U^{i} V^{j} W^{k}, \\
\frac{d W}{d t}=\varepsilon W+\sum_{i+j+k=2} c_{i j k} U^{i} V^{j} W^{k},
\end{gathered}
$$

where $a_{i j k}, b_{i j k}$, and $c_{i j k}$ for $i, j, k=0,1,2$ are functions of the parameters $\beta_{i}$ and $\alpha_{i j}$ in system (1), $u$ and $v>0$ are bounded parameters, and $0<\varepsilon \ll 1$ is perturbation parameter. And then we get the real normal form of the system (2) after a series of transformations. Two examples are provided to illustrate these results in the last section.

\section{Preliminary Theorems}

In this section, we first consider a normal form system of degree two in $\mathbb{R}^{3}$. Then, by a series of transformations we introduce some theorems for the normal form. The reader is referred to [15] for more details about the following content.

Consider the 3-dimensional system

$$
\dot{x}=D x+X_{2}(x),
$$

where $X_{2}(x)=O\left(|x|^{2}\right)$ is $C^{\infty}$ in $x \in \mathbb{R}^{3}$, and

$$
D=\left(\begin{array}{ccc}
0 & 1 & 0 \\
-1 & 0 & 0 \\
0 & 0 & 0
\end{array}\right)
$$

By adding up the 2-parameter linear part $\operatorname{diag}\left(\lambda_{1}, \lambda_{1}, \lambda_{2}\right) x$ we obtain

$$
\dot{x}=D\left(\lambda_{1}, \lambda_{2}\right) x+X_{2}(x),
$$

where $D\left(\lambda_{1}, \lambda_{2}\right)=\operatorname{diag}\left(A\left(\lambda_{1}\right), \lambda_{2}\right)$, with

$$
A\left(\lambda_{1}\right)=\left(\begin{array}{cc}
\lambda_{1} & 1 \\
-1 & \lambda_{1}
\end{array}\right) \text {. }
$$

It can be verified that (5) has the following real normal form up to order 3 (see [15]):

$$
\begin{aligned}
\dot{x}_{1}= & \lambda_{1} x_{1}+x_{2}+a_{1} x_{1} x_{3}+b_{1} x_{2} x_{3}+\left(a_{2} x_{1}+b_{2} x_{2}\right) \\
& \times\left(x_{1}^{2}+x_{2}^{2}\right)+\left(a_{3} x_{1}+b_{3} x_{2}\right) x_{3}^{2}+O\left(\left|x_{1}, x_{2}, x_{3}\right|^{4}\right), \\
\dot{x}_{2}= & -x_{1}+\lambda_{1} x_{2}-b_{1} x_{1} x_{3}+a_{1} x_{2} x_{3}+\left(-b_{2} x_{1}+a_{2} x_{2}\right) \\
& \times\left(x_{1}^{2}+x_{2}^{2}\right)+\left(-b_{3} x_{1}+a_{3} x_{2}\right) x_{3}^{2}+O\left(\left|x_{1}, x_{2}, x_{3}\right|^{4}\right), \\
\dot{x}_{3}= & \lambda_{2} x_{3}+c_{1}\left(x_{1}^{2}+x_{2}^{2}\right)+d_{1} x_{3}^{2}+c_{2}\left(x_{1}^{2}+x_{2}^{2}\right) x_{3} \\
& +d_{2} x_{3}^{3}+O\left(\left|x_{1}, x_{2}, x_{3}\right|^{4}\right) .
\end{aligned}
$$

For convenience, we assume that $a_{1} b_{1} c_{1} \neq 0$ as in [15]. By the scaling

$x_{1} \rightarrow \frac{\sqrt{\left|d_{1}\right|}}{\sqrt{\left|c_{1}\right|} a_{1}} x_{1}, \quad x_{2} \rightarrow \frac{\sqrt{\left|d_{1}\right|}}{\sqrt{\left|c_{1}\right|} a_{1}} x_{2}, \quad x_{3} \rightarrow \frac{1}{a_{1}} x_{3}$,
(7) becomes

$$
\begin{aligned}
\dot{x}_{1}= & \lambda_{1} x_{1}+x_{2}+x_{1} x_{3}+\widetilde{b}_{1} x_{2} x_{3}+\left(\tilde{a}_{2} x_{1}+\widetilde{b}_{2} x_{2}\right)\left(x_{1}^{2}+x_{2}^{2}\right) \\
& +\left(\widetilde{a}_{3} x_{1}+\widetilde{b}_{3} x_{2}\right) x_{3}^{2}+O\left(\left|x_{1}, x_{2}, x_{3}\right|^{4}\right), \\
\dot{x}_{2}= & -x_{1}+\lambda_{1} x_{2}-\widetilde{b}_{1} x_{1} x_{3}+x_{2} x_{3}+\left(-\tilde{b}_{2} x_{1}+\tilde{a}_{2} x_{2}\right) \\
& \times\left(x_{1}^{2}+x_{2}^{2}\right)+\left(-\tilde{b}_{3} x_{1}+\tilde{a}_{3} x_{2}\right) x_{3}^{2}+O\left(\left|x_{1}, x_{2}, x_{3}\right|^{4}\right), \\
\dot{x}_{3}= & \lambda_{2} x_{3}+\widetilde{c}_{1}\left(x_{1}^{2}+x_{2}^{2}\right)+\tilde{d}_{1} x_{3}^{2}+\widetilde{c}_{2}\left(x_{1}^{2}+x_{2}^{2}\right) x_{3} \\
& +\tilde{d}_{2} x_{3}^{3}+O\left(\left|x_{1}, x_{2}, x_{3}\right|^{4}\right),
\end{aligned}
$$

where

$$
\begin{gathered}
\tilde{b}_{1}=\frac{b_{1}}{a_{1}}, \quad \tilde{a}_{2}=\frac{\left|d_{1}\right| a_{2}}{\left|c_{1}\right| a_{1}^{2}}, \quad \tilde{b}_{2}=\frac{\left|d_{1}\right| b_{2}}{\left|c_{1}\right| a_{1}^{2}}, \\
\tilde{a}_{3}=\frac{a_{3}}{a_{1}^{2}}, \quad \tilde{b}_{3}=\frac{b_{3}}{a_{1}^{2}}, \quad \tilde{c}_{1}=\frac{\left|d_{1}\right|}{a_{1}} \operatorname{sgn}\left(c_{1}\right), \\
\tilde{d}_{1}=\frac{d_{1}}{a_{1}}, \quad \tilde{c}_{2}=\frac{\left|d_{1}\right| c_{2}}{\left|c_{1}\right| a_{1}^{2}}, \quad \tilde{d}_{2}=\frac{d_{2}}{a_{1}^{2}} .
\end{gathered}
$$

Then, by introducing polar coordinates

$$
x_{1}=p \cos \theta, \quad x_{2}=-p \sin \theta,
$$

(9) further becomes

$$
\begin{gathered}
\dot{\theta}=1+\widetilde{b}_{1} x_{3}+\widetilde{b}_{2} p^{2}+\widetilde{b}_{3} x_{3}^{2}+p^{-1} S_{1}\left(\theta, p, x_{3}\right), \\
\dot{p}=\lambda_{1} p+p x_{3}+\widetilde{a}_{2} p^{3}+\widetilde{a}_{3} p x_{3}^{2}+S_{2}\left(\theta, p, x_{3}\right), \\
\dot{x}_{3}=\lambda_{2} x_{3}+\widetilde{c}_{1} p^{2}+\widetilde{d}_{1} x_{3}^{2}+\widetilde{c}_{2} p^{2} x_{3}+\widetilde{d}_{2} x_{3}^{3}+S_{3}\left(\theta, p, x_{3}\right),
\end{gathered}
$$

where $S_{1}, S_{2}$, and $S_{3}$ are $2 \pi$ periodic in $\theta$, and $S_{1}, S_{2}$, and $S_{3}=$ $\mathrm{O}\left(\left|p, x_{3}\right|^{4}\right)$. By a further scaling of the form

$$
\begin{array}{r}
p \longrightarrow \varepsilon p, \quad x_{3} \longrightarrow \varepsilon x_{3}, \\
\lambda_{1} \longrightarrow \varepsilon \delta_{1}, \quad \lambda_{2} \longrightarrow \varepsilon \delta_{2}, \\
\varepsilon>0,\left|\delta_{1}\right|=1,
\end{array}
$$

(12) becomes

$$
\begin{gathered}
\dot{\theta}=1+\varepsilon\left[\widetilde{b}_{1} x_{3}+\varepsilon\left(\widetilde{b}_{2} p^{2}+\widetilde{b}_{3} x_{3}^{2}\right)\right]+p^{-1} \varepsilon^{3} O\left(\left|p, x_{3}\right|^{4}\right), \\
\dot{p}=\varepsilon p\left[\delta_{1}+x_{3}+\varepsilon\left(\widetilde{a}_{2} p^{2}+\widetilde{a}_{3} x_{3}^{2}\right)\right]+O\left(\varepsilon^{3}\right), \\
\dot{x}_{3}=\varepsilon\left[\delta_{2} x_{3}+\widetilde{c}_{1} p^{2}+\widetilde{d}_{1} x_{3}^{2}+\varepsilon\left(\widetilde{c}_{2} p^{2} x_{3}+\widetilde{d}_{2} x_{3}^{3}\right)\right]+O\left(\varepsilon^{3}\right) .
\end{gathered}
$$

We obtain from (14)

$$
\begin{aligned}
& \frac{d p}{d \theta}=\varepsilon p\left[f_{0}\left(p, x_{3}\right)+\varepsilon f_{1}\left(p, x_{3}\right)+\bar{f}\left(\theta, p, x_{3}, \varepsilon\right)\right], \\
& \frac{d x_{3}}{d \theta}=\varepsilon\left[g_{0}\left(p, x_{3}\right)+\varepsilon g_{1}\left(p, x_{3}\right)+\bar{g}\left(\theta, p, x_{3}, \varepsilon\right)\right],
\end{aligned}
$$


where

$$
\begin{gathered}
f_{0}\left(p, x_{3}\right)=\delta_{1}+x_{3}, \\
g_{0}\left(p, x_{3}\right)=\delta_{2} x_{3}+\widetilde{c}_{1} p^{2}+\widetilde{d}_{1} x_{3}^{2}, \\
f_{1}\left(p, x_{3}\right)=-\delta_{1} \widetilde{b}_{1} x_{3}+\widetilde{a}_{2} p^{2}+\left(\widetilde{a}_{3}-\widetilde{b}_{1}\right) x_{3}^{2}, \\
g_{1}\left(p, x_{3}\right)=-\widetilde{b}_{1} \delta_{2} x_{3}^{2}+\left(\widetilde{c}_{2}-\widetilde{c}_{1} \widetilde{b}_{1}\right) p^{2} x_{3}+\left(\widetilde{d}_{2}-\widetilde{b}_{1} \widetilde{d}_{1}\right) x_{3}^{3}, \\
\bar{f}, \bar{g}=O\left(\varepsilon^{2}\right) .
\end{gathered}
$$

Note that the functions $\bar{f}$ and $\bar{g}$ in (15) are $2 \pi$ periodic in $\theta$ but may not be well defined at $p=0$. Thus, we suppose $p \gg \varepsilon>0$ for (15).

The averaging system

$$
\begin{aligned}
& \frac{d p}{d \theta}=p f_{0}\left(p, x_{3}\right), \\
& \frac{d x_{3}}{d \theta}=g_{0}\left(p, x_{3}\right)
\end{aligned}
$$

has a singular point $\left(p_{0}, s_{0}\right)$ on the half plane $p>0$ if

$$
\widetilde{c}_{1}\left(\widetilde{d}_{1}-\delta_{1} \delta_{2}\right)<0
$$

where $p_{0}=\sqrt{\left(\delta_{1} \delta_{2}-\widetilde{d}_{1}\right) / \widetilde{c}_{1}}, s_{0}=-\delta_{1}$. By denoting

$$
B=\left.\frac{\partial\left(p f_{0}, g_{0}\right)}{\partial\left(p, x_{3}\right)}\right|_{\left(p_{0}, s_{0}\right)},
$$

we obtain $|B|=-2 \widetilde{c}_{1} p_{0}^{2} \neq 0$, and the characteristic polynomial of $B$ is $f_{\lambda}(B)=\lambda^{2}-\left(\delta_{2}-2 \widetilde{d}_{1} \delta_{1}\right) \lambda-2\left(\widetilde{d}_{1}-\delta_{1} \delta_{2}\right)$. We define

$$
\Delta=\left(\delta_{2}-2 \tilde{d}_{1} \delta_{1}\right)^{2}+8\left(\tilde{d}_{1}-\delta_{1} \delta_{2}\right) .
$$

According to Theorem 4.1.3 in [15], we can obtain the following theorem.

Theorem 1. Suppose that (18) holds. Then, (7) has a periodic orbit near the origin for $0<\varepsilon \ll 1$. Further, the periodic orbit is stable (resp. unstable) if one (resp. none) of the following conditions holds:

(a) $\Delta=0$ and $\delta_{2}-2 \widetilde{d}_{1} \delta_{1}<0$,

(b) $\Delta<0$ and $\delta_{2}-2 \widetilde{d}_{1} \delta_{1} \leq 0$,

(c) $\Delta>0, \delta_{2}-2 \widetilde{d}_{1} \delta_{1}<0$, and $\widetilde{d}_{1}-\delta_{1} \delta_{2}<0$,

where $\Delta$ is given by (20).

Then, by letting $s=x_{3}+\delta_{1}$ and $\theta \rightarrow \varepsilon^{-1} \theta$ and truncating the terms of order $\varepsilon^{2}$, we have from (15)

$$
\begin{aligned}
& \frac{d p}{d \theta}=p s+\varepsilon p\left[\tilde{a}_{2} p^{2}+f_{2}(s)\right], \\
& \frac{d s}{d \theta}=\widetilde{c}_{1} p^{2}+g_{2}(s)+\varepsilon\left[g_{3}(s) p^{2}+g_{4}(s)\right],
\end{aligned}
$$

where

$$
\begin{aligned}
& f_{2}(s)=\left(\widetilde{a}_{3}-\widetilde{b}_{1}\right) s^{2}+\delta_{1}\left(\widetilde{b}_{1}-2 \widetilde{a}_{3}\right) s+\widetilde{a}_{3}, \\
& g_{2}(s)=\widetilde{d}_{1} s^{2}+\left(\delta_{2}-2 \delta_{1} \widetilde{d}_{1}\right) s+\delta_{1}\left(\delta_{1} \widetilde{d}_{1}-\delta_{2}\right), \\
& g_{3}(s)=\left(\widetilde{c}_{2}-\widetilde{c}_{1} \widetilde{b}_{1}\right)\left(s-\delta_{1}\right), \\
& g_{4}(s)=\left(\widetilde{d}_{2}-\widetilde{b}_{1} \widetilde{d}_{1}\right)\left(s-\delta_{1}\right)^{3}-\delta_{2} \widetilde{b}_{1}\left(s-\delta_{1}\right)^{2} .
\end{aligned}
$$

Thus, in order that (21) has a limit cycle, we necessarily suppose

$$
\delta_{2}-2 \delta_{1} \widetilde{d}_{1}=\delta \varepsilon, \quad \delta \in \mathbb{R},
$$

and $\widetilde{c}_{1}<0, \widetilde{c}_{1} \widetilde{d}_{1}>0$, that is,

$$
a_{1} c_{1}<0, \quad c_{1} d_{1}>0 .
$$

This yields $\widetilde{c}_{1}=\widetilde{d}_{1}<0$, and hence (21) becomes

$$
\begin{aligned}
& \frac{d p}{d \theta}=p s+\varepsilon p\left[\widetilde{a}_{2} p^{2}+f_{2}(s)\right], \\
& \frac{d s}{d \theta}=\widetilde{c}_{1}\left(p^{2}+s^{2}-1\right)+\varepsilon\left[g_{3}(s) p^{2}+\bar{g}_{4}(s)+O(\varepsilon)\right],
\end{aligned}
$$

where

$$
\begin{aligned}
\bar{g}_{4}(s)= & \left(\widetilde{d}_{2}-\widetilde{c}_{1} \widetilde{b}_{1}\right) s^{3}+\delta_{1}\left(\widetilde{c}_{1} \widetilde{b}_{1}-3 \widetilde{d}_{2}\right) s^{2} \\
& +\left(\delta+3 \widetilde{d}_{2}+\widetilde{c}_{1} \widetilde{b}_{1}\right) s-\left(\delta+\widetilde{d}_{2}+\widetilde{c}_{1} \widetilde{b}_{1}\right) \delta_{1} .
\end{aligned}
$$

For small $\varepsilon>0$, (25) has a focus $A_{\varepsilon}(p(\varepsilon), s(\varepsilon))$ with

$$
p(0)=1, \quad s(0)=0, \quad s^{\prime}(0)=-\left(\widetilde{a}_{3}+\widetilde{a}_{2}\right) .
$$

We define

$$
\begin{aligned}
\delta_{0}=2 \widetilde{c}_{1} \widetilde{a}_{3}-3 \widetilde{d}_{2}-\widetilde{c}_{2}-2 \widetilde{a}_{2}\left(1-\widetilde{c}_{1}\right), \\
\Delta_{0}=\frac{1}{8}\left[3 \widetilde{d}_{2}-\widetilde{c}_{2}-2 \widetilde{a}_{2}\left(1-\widetilde{c}_{1}\right)-2 \widetilde{c}_{1} \widetilde{a}_{3}\right], \\
\delta_{0}^{\prime}=\frac{2}{3-2 \widetilde{c}_{1}}\left[\left(2-\widetilde{c}_{1}\right)\left(2 \widetilde{c}_{1} \widetilde{a}_{3}-3 \widetilde{d}_{2}\right)-\left(1-\widetilde{c}_{1}\right)\right. \\
\left.\quad \times\left(\widetilde{c}_{2}+2 \widetilde{a}_{2}\left(1-\widetilde{c}_{1}\right)\right)\right] .
\end{aligned}
$$

By using the coefficients in (7), we have

$$
\begin{aligned}
& \delta_{0}=\frac{2 d_{1} a_{3}}{a_{1}^{3}}-\frac{3 d_{2}}{a_{1}^{2}}-\frac{d_{1}}{c_{1} a_{1}^{2}}\left[c_{2}+2 a_{2}\left(1-\frac{d_{1}}{a_{1}}\right)\right], \\
& \Delta_{0}=\frac{1}{8}\left[\frac{1}{a_{1}^{3}}\left(3 a_{1} d_{2}-2 a_{3} d_{1}\right)\right. \\
& \left.\quad-\frac{d_{1}}{c_{1} a_{1}^{2}}\left(c_{2}+2 a_{2}\left(1-\frac{d_{1}}{a_{1}}\right)\right)\right], \\
& \begin{aligned}
\delta_{0}^{\prime}=\frac{2}{3 a_{1}-2 d_{1}} & {\left[\left(2-\frac{d_{1}}{a_{1}}\right)\left(\frac{2 d_{1} a_{3}}{a_{1}^{2}}-\frac{3 d_{2}}{a_{1}}\right)\right.} \\
& \left.\quad-\frac{d_{1}}{c_{1} a_{1}}\left(1-\frac{d_{1}}{a_{1}}\right)\left(c_{2}+2 a_{2}\left(1-\frac{d_{1}}{a_{1}}\right)\right)\right] .
\end{aligned}
\end{aligned}
$$

Then, in 1997, the following result was obtained in [15]. 
Theorem 2. Suppose that (24) holds and $\Delta_{0} \neq 0$. Then, for any given $\varepsilon_{1}>0$ there exist an $\varepsilon_{0}>0$ and a $C^{1}$ function $\phi_{0}\left(\lambda_{1}\right)=$ $\left(2 d_{1} \lambda_{1} / a_{1}\right)+\delta_{0} \lambda_{1}^{2}+O\left(\lambda_{1}^{3}\right)$ and $\phi_{1}\left(\lambda_{1}\right)=\left(2 d_{1} \lambda_{1} / a_{1}\right)+\delta_{0}^{\prime} \lambda_{1}^{2}+$ $O\left(\lambda_{1}^{3}\right)$ such that for $0<\lambda_{1}^{2}+\lambda_{2}^{2}<\varepsilon_{0}$, (7) has a unique invariant torus near the origin if $\Delta_{0} \phi_{1}\left(\lambda_{1}\right)-\varepsilon_{1} \lambda_{1}^{2}<\Delta_{0} \lambda_{2}<\Delta_{0} \phi_{0}\left(\lambda_{1}\right)$ and has no invariant torus if $\Delta_{0} \lambda_{2}>\Delta_{0} \phi_{0}\left(\lambda_{1}\right)$. Moreover, the torus, if it exists, is stable (resp. unstable) when $\Delta_{0}<0$ (resp. > 0).

\section{Normal Form of System (2)}

In this section, we consider system (1) in the first octant $\mathbb{R}_{+}^{3}$, where $\mathbb{R}_{+}=\{x \in \mathbb{R}: x>0\}$. We now look for the conditions for the existence of positive equilibria of system (1), which is equivalent to find the positive solutions of the following system:

$$
\beta_{i}+\sum_{j=1}^{3} \alpha_{i j} X_{j}=0, \quad i=1,2,3 .
$$

We suppose that there exists at least one positive solution of (30). Without loss of generality, we assume that the positive equilibrium is $(1,1,1)$. Then, we move it to the origin by doing the change of variables $Y_{i}=X_{i}-1, i=1,2,3$. Then, system (1) can be written as

$$
\frac{d Y_{i}}{d t}=\left(Y_{i}+1\right) \sum_{j=1}^{3} \alpha_{i j} Y_{j}, \quad i=1,2,3 .
$$

Now, we shall investigate a special form of system (31) with a small parameter; we write the perturbed system as

$$
\frac{d Y_{i}}{d t}=\left(Y_{i}+1\right) \sum_{j=1}^{3} \alpha_{i j}(\varepsilon) Y_{j}, \quad i=1,2,3 .
$$

Denote $M(\varepsilon)=\left(\alpha_{i j}(\varepsilon)\right)_{3 \times 3}$, and we suppose $M(\varepsilon)$ is similar to

$$
\Psi=\left(\begin{array}{ccc}
u \varepsilon & v & 0 \\
-v & u \varepsilon & 0 \\
0 & 0 & \varepsilon
\end{array}\right), \quad(u, v)=(u(\varepsilon), v(\varepsilon)) .
$$

Then, system (32) can be changed into the system (2) by a linear transformation.

In this section, our task is to change system (2) into the normal form of (7). Making the transformation

$$
x_{1}=U, \quad x_{2}=V, \quad x_{3}=W, \quad t \longrightarrow \frac{1}{v} t,
$$

system (2) becomes

$$
\begin{aligned}
& \dot{x}_{1}=\lambda_{1} x_{1}+x_{2}+\sum_{i+j+k=2} \widetilde{a}_{i j k} x_{1}^{i} x_{2}^{j} x_{3}^{k}, \\
& \dot{x}_{2}=-x_{1}+\lambda_{1} x_{2}+\sum_{i+j+k=2} \widetilde{b}_{i j k} x_{1}^{i} x_{2}^{j} x_{3}^{k}, \\
& \dot{x}_{3}=\lambda_{2} x_{3}+\sum_{i+j+k=2} \widetilde{c}_{i j k} x_{1}^{i} x_{2}^{j} x_{3}^{k},
\end{aligned}
$$

where

$$
\begin{gathered}
\tilde{a}_{i j k}=\frac{1}{v} a_{i j k}, \quad \widetilde{b}_{i j k}=\frac{1}{v} b_{i j k}, \quad \tilde{c}_{i j k}=\frac{1}{v} c_{i j k}, \\
\lambda_{1}=\frac{u}{v} \varepsilon, \quad \lambda_{2}=\frac{1}{v} \varepsilon .
\end{gathered}
$$

Let

$$
T=\left(\begin{array}{lll}
1 & i & 0 \\
i & 1 & 0 \\
0 & 0 & 1
\end{array}\right),
$$

by changing $y=T x$, where $y=\left(y_{1}, y_{2}, y_{3}\right)^{T}, x=$ $\left(x_{1}, x_{2}, x_{3}\right)^{T}$, and system (35) becomes a complex system of the form

$$
\begin{gathered}
\dot{y}_{1}=\left(\lambda_{1}+i\right) y_{1}+\sum_{i+j+k=2} a_{i j k}^{*} y_{1}^{i} y_{2}^{j} y_{3}^{k}, \\
\dot{y}_{2}=\left(\lambda_{1}-i\right) y_{2}+\sum_{i+j+k=2} b_{i j k}^{*} y_{1}^{i} y_{2}^{j} y_{3}^{k}, \\
\dot{y}_{3}=\lambda_{2} y_{3}+\sum_{i+j+k=2} c_{i j k}^{*} y_{1}^{i} y_{2}^{j} y_{3}^{k},
\end{gathered}
$$

where

$$
\begin{aligned}
& a_{200}^{*}=\frac{1}{4}\left(\widetilde{a}_{200}-\widetilde{a}_{020}+\widetilde{b}_{110}\right)+\frac{1}{4}\left(-\widetilde{a}_{110}+\widetilde{b}_{200}-\widetilde{b}_{020}\right) i, \\
& a_{020}^{*}=\frac{1}{4}\left(\widetilde{a}_{020}-\widetilde{a}_{200}+\widetilde{b}_{110}\right)+\frac{1}{4}\left(-\widetilde{a}_{110}+\widetilde{b}_{020}-\widetilde{b}_{200}\right) i, \\
& a_{002}^{*}=\widetilde{a}_{002}+\widetilde{b}_{002} i, \\
& a_{110}^{*}=\frac{1}{2}\left(\widetilde{b}_{200}+\widetilde{b}_{020}\right)-\frac{1}{2}\left(\widetilde{a}_{200}+\widetilde{a}_{020}\right) i, \\
& a_{101}^{*}=\frac{1}{2}\left(\widetilde{a}_{101}-\widetilde{a}_{011}\right)+\frac{1}{2}\left(\widetilde{b}_{101}-\widetilde{b}_{011}\right) i, \\
& a_{011}^{*}=\frac{1}{2}\left(\widetilde{a}_{011}+\widetilde{b}_{101}\right)+\frac{1}{2}\left(-\widetilde{a}_{101}+\widetilde{b}_{011}\right) i, \\
& b_{200}^{*}=\frac{1}{4}\left(\widetilde{b}_{200}-\widetilde{b}_{020}+\widetilde{a}_{110}\right)+\frac{1}{4}\left(-\widetilde{b}_{110}+\widetilde{a}_{200}-\widetilde{a}_{020}\right) i, \\
& b_{020}^{*}=\frac{1}{4}\left(\widetilde{b}_{020}-\widetilde{b}_{200}+\widetilde{a}_{110}\right)+\frac{1}{4}\left(-\widetilde{b}_{110}+\widetilde{a}_{020}-\widetilde{a}_{200}\right) i, \\
& b_{002}^{*}=\widetilde{b}_{002}+\widetilde{a}_{002} i, \\
& b_{110}^{*}=\frac{1}{2}\left(\widetilde{a}_{200}+\widetilde{a}_{020}\right)-\frac{1}{2}\left(\widetilde{b}_{200}+\widetilde{b}_{020}\right) i, \\
& b_{101}^{*}=\frac{1}{2}\left(\widetilde{b}_{101}-\widetilde{b}_{011}\right)+\frac{1}{2}\left(\widetilde{a}_{101}-\widetilde{a}_{011}\right) i, \\
& b_{011}^{*}=\frac{1}{2}\left(\widetilde{b}_{011}+\widetilde{a}_{101}\right)+\frac{1}{2}\left(-\widetilde{b}_{101}+\widetilde{a}_{011}\right) i, \\
& c_{200}^{*}=\frac{1}{4}\left(\widetilde{a}_{200}-\widetilde{a}_{020}\right)-\frac{1}{4} \widetilde{a}_{110} i, \\
& c_{020}^{*}=\frac{1}{4}\left(\widetilde{a}_{020}-\widetilde{a}_{200}\right)-\frac{1}{4} \widetilde{a}_{110} i,
\end{aligned}
$$




$$
\begin{aligned}
& c_{002}^{*}=\widetilde{a}_{002}, \\
& c_{110}^{*}=-\frac{1}{2}\left(\tilde{a}_{200}+\tilde{a}_{020}\right) i, \\
& c_{101}^{*}=\frac{1}{2}\left(\tilde{a}_{101}-\tilde{a}_{011}\right), \\
& c_{011}^{*}=\frac{1}{2} \widetilde{a}_{011}-\frac{1}{2} \widetilde{a}_{101} i .
\end{aligned}
$$

By the fundamental theory of normal form [16], we know that system (38) can be converted to the normal form by some transformations. So our following task is to find the transformations and work out the normal form of system (38).

We denote (38) as $\dot{y}=F(y)$, where $F(0)=0$, and for simplicity, we write the nonlinear part of (38) as $\Theta(y)$. By doing the following transformation:

$$
y=z+P(z) \equiv h_{1}(z), \quad z \in \mathbb{R}^{3}
$$

where $P(z)=\left(P_{1}(z), P_{2}(z), P_{3}(z)\right)^{T}$, which is to be determined, (38) becomes

$$
\dot{z}=\left[D h_{1}(z)\right]^{-1} F\left(h_{1}(z)\right) \text {. }
$$

Then, by noting

$$
\left(D h_{1}\right)^{-1}=I-D P+(D P)^{2}+O\left(|D P|^{3}\right)
$$

we can get from (41)

$$
\begin{aligned}
\dot{z}_{i}= & \gamma_{i} z_{i}+\gamma_{i} P_{i}-\sum_{j=1}^{3} \frac{\partial P_{i}}{\partial z_{j}} \gamma_{j} z_{j} \\
& +\Theta_{i}(z)+O\left(|z|^{3}\right), \quad i=1,2,3,
\end{aligned}
$$

where $\gamma_{1}=\lambda_{1}+i$ and $\gamma_{2}=\lambda_{1}-i, \gamma_{3}=\lambda_{2}$. In order to eliminate the quadratic homogeneous polynomial, we need

$$
\gamma_{i} P_{i}-\sum_{j=1}^{3} \frac{\partial P_{i}}{\partial z_{j}} \gamma_{j} z_{j}=-\Theta_{i}(z)+O\left(|z|^{3}\right), \quad i=1,2,3
$$

We take $P_{i}, i=1,2,3$ as quadratic homogeneous polynomial, having the form

$$
P_{i}=l_{i 1} z_{1}^{2}+l_{i 2} z_{2}^{2}+l_{i 3} z_{3}^{2}+l_{i 4} z_{1} z_{2}+l_{i 5} z_{1} z_{3}+l_{i 6} z_{2} z_{3} \text {, }
$$

where $l_{i k}, k=1, \ldots, 6$, are real undermined coefficients. By inserting (45) into (44) and comparing the coefficients of similar items, we can obtain

$$
\begin{aligned}
& l_{11}=\frac{a_{200}^{*}}{\gamma_{1}}, \quad l_{12}=\frac{a_{020}^{*}}{2 \gamma_{2}-\gamma_{1}}, \quad l_{13}=\frac{a_{002}^{*}}{2 \gamma_{3}-\gamma_{1}} \\
& l_{14}=\frac{a_{110}^{*}}{\gamma_{2}}, \quad l_{15}=\frac{a_{101}^{*}}{\gamma_{3}}, \quad l_{16}=\frac{a_{200}^{*}}{\gamma_{2}+\gamma_{3}-\gamma_{1}} \text {; } \\
& l_{21}=\frac{b_{200}^{*}}{2 \gamma_{1}-\gamma_{2}}, \quad l_{22}=\frac{b_{020}^{*}}{\gamma_{2}}, \quad l_{23}=\frac{b_{002}^{*}}{2 \gamma_{3}-\gamma_{2}}, \\
& l_{24}=\frac{b_{110}^{*}}{\gamma_{1}}, \quad l_{25}=\frac{b_{101}^{*}}{\gamma_{1}+\gamma_{3}-\gamma_{2}}, \quad l_{26}=\frac{a_{200}^{*}}{\gamma_{3}} \text {; } \\
& l_{31}=\frac{c_{200}^{*}}{2 \gamma_{1}-\gamma_{3}}, \quad l_{32}=\frac{c_{020}^{*}}{2 \gamma_{2}-\gamma_{3}}, \quad l_{33}=\frac{c_{002}^{*}}{\gamma_{3}} \text {, } \\
& l_{34}=\frac{c_{110}^{*}}{\gamma_{1}+\gamma_{2}-\gamma_{3}}, \quad l_{35}=\frac{c_{101}^{*}}{\gamma_{1}}, \quad l_{36}=\frac{c_{200}^{*}}{\gamma_{2}} \text {. }
\end{aligned}
$$

Note that $\left|\gamma_{3}\right|=\left|\lambda_{2}\right| \ll 1,\left|\gamma_{1}+\gamma_{2}-\gamma_{3}\right|=\left|2 \lambda_{1}-\lambda_{2}\right| \ll 1$. The terms with coefficients $l_{15}, l_{26}, l_{33}$, and $l_{34}$ that appeared above cannot be removed. Those terms are called the resonance terms. Then, we have

$$
\begin{aligned}
& P_{1}=l_{11} z_{1}^{2}+l_{12} z_{2}^{2}+l_{13} z_{3}^{2}+l_{14} z_{1} z_{2}+l_{16} z_{2} z_{3}, \\
& P_{2}=l_{21} z_{1}^{2}+l_{22} z_{2}^{2}+l_{23} z_{3}^{2}+l_{24} z_{1} z_{2}+l_{25} z_{1} z_{3}, \\
& P_{3}=l_{31} z_{1}^{2}+l_{32} z_{2}^{2}+l_{35} z_{1} z_{3}+l_{36} z_{2} z_{3},
\end{aligned}
$$

and system (43) becomes

$$
\begin{aligned}
& \dot{z}_{1}=\gamma_{1} z_{1}+a_{101}^{*} z_{1} z_{3}+O\left(\left|z_{1}, z_{2}, z_{3}\right|^{3}\right), \\
& \dot{z}_{2}=\gamma_{2} z_{2}+b_{011}^{*} z_{2} z_{3}+O\left(\left|z_{1}, z_{2}, z_{3}\right|^{3}\right), \\
& \dot{z}_{3}=\gamma_{3} z_{3}+c_{002}^{*} z_{3}^{2}+c_{110}^{*} z_{1} z_{2}+O\left(\left|z_{1}, z_{2}, z_{3}\right|^{3}\right) .
\end{aligned}
$$

Let $L(z)$ denote the cubic terms in $z$ of (48). Then, from (41) and (42) we have

$$
L(z)=\left(\begin{array}{c}
-\sum_{j=1}^{3} P_{1 j} \gamma_{j} P_{j} \\
-\sum_{j=1}^{3} P_{2 j} \gamma_{j} P_{j} \\
-\sum_{j=1}^{3} P_{3 j} \gamma_{j} P_{j}
\end{array}\right)+\left(\begin{array}{l}
h_{1} \\
h_{2} \\
h_{3}
\end{array}\right)+(D P)^{2}\left(\begin{array}{l}
\gamma_{1} z_{1} \\
\gamma_{2} z_{2} \\
\gamma_{3} z_{3}
\end{array}\right),
$$


where $P_{i j}=\partial P_{i} / \partial z_{j}, i, j=1,2,3$,

$$
\begin{aligned}
h_{1}= & -P_{11}\left(a_{200}^{*} z_{1}^{2}+a_{020}^{*} z_{2}^{2}+a_{002}^{*} z_{3}^{2}\right) \\
& -P_{12}\left(b_{200}^{*} z_{1}^{2}+b_{020}^{*} z_{2}^{2}+b_{002}^{*} z_{3}^{2}\right) \\
& -P_{13}\left(c_{200}^{*} z_{1}^{2}+c_{020}^{*} z_{2}^{2}+c_{002}^{*} z_{3}^{2}\right) \\
& -P_{11}\left(a_{110}^{*} z_{1} z_{2}+a_{101}^{*} z_{1} z_{3}+a_{011}^{*} z_{2} z_{3}\right) \\
& -P_{12}\left(b_{110}^{*} z_{1} z_{2}+b_{101}^{*} z_{1} z_{3}+b_{011}^{*} z_{2} z_{3}\right) \\
& -P_{13}\left(c_{110}^{*} z_{1} z_{2}+c_{101}^{*} z_{1} z_{3}+c_{011}^{*} z_{2} z_{3}\right) \\
& +2\left(a_{200}^{*} z_{1} P_{1}+a_{020}^{*} z_{2} P_{2}+a_{002}^{*} z_{3} P_{3}\right) \\
& +a_{110}^{*}\left(z_{1} P_{2}+z_{2} P_{1}\right)+a_{101}^{*}\left(z_{1} P_{3}+z_{3} P_{1}\right) \\
& +a_{011}^{*}\left(z_{2} P_{3}+z_{3} P_{2}\right) \\
h_{2}= & -P_{21}\left(a_{200}^{*} z_{1}^{2}+a_{020}^{*} z_{2}^{2}+a_{002}^{*} z_{3}^{2}\right) \\
& -P_{22}\left(b_{200}^{*} z_{1}^{2}+b_{020}^{*} z_{2}^{2}+b_{002}^{*} z_{3}^{2}\right) \\
& -P_{23}\left(c_{200}^{*} z_{1}^{2}+c_{020}^{*} z_{2}^{2}+c_{002}^{*} z_{3}^{2}\right) \\
& -P_{21}\left(a_{110}^{*} z_{1} z_{2}+a_{101}^{*} z_{1} z_{3}+a_{011}^{*} z_{2} z_{3}\right)
\end{aligned}
$$

$$
\begin{aligned}
& -P_{22}\left(b_{110}^{*} z_{1} z_{2}+b_{101}^{*} z_{1} z_{3}+b_{011}^{*} z_{2} z_{3}\right) \\
& -P_{23}\left(c_{110}^{*} z_{1} z_{2}+c_{101}^{*} z_{1} z_{3}+c_{011}^{*} z_{2} z_{3}\right) \\
& +2\left(b_{200}^{*} z_{1} P_{1}+b_{020}^{*} z_{2} P_{2}+b_{002}^{*} z_{3} P_{3}\right) \\
& +b_{110}^{*}\left(z_{1} P_{2}+z_{2} P_{1}\right)+b_{101}^{*}\left(z_{1} P_{3}+z_{3} P_{1}\right) \\
& +b_{011}^{*}\left(z_{2} P_{3}+z_{3} P_{2}\right) \\
h_{3}= & -P_{31}\left(a_{200}^{*} z_{1}^{2}+a_{020}^{*} z_{2}^{2}+a_{002}^{*} z_{3}^{2}\right) \\
& -P_{32}\left(b_{200}^{*} z_{1}^{2}+b_{020}^{*} z_{2}^{2}+b_{002}^{*} z_{3}^{2}\right) \\
& -P_{33}\left(c_{200}^{*} z_{1}^{2}+c_{020}^{*} z_{2}^{2}+c_{002}^{*} z_{3}^{2}\right) \\
& -P_{31}\left(a_{110}^{*} z_{1} z_{2}+a_{101}^{*} z_{1} z_{3}+a_{011}^{*} z_{2} z_{3}\right) \\
& -P_{32}\left(b_{110}^{*} z_{1} z_{2}+b_{101}^{*} z_{1} z_{3}+b_{011}^{*} z_{2} z_{3}\right) \\
& -P_{33}\left(c_{110}^{*} z_{1} z_{2}+c_{101}^{*} z_{1} z_{3}+c_{011}^{*} z_{2} z_{3}\right) \\
& +2\left(c_{200}^{*} z_{1} P_{1}+c_{020}^{*} z_{2} P_{2}+c_{002}^{*} z_{3} P_{3}\right) \\
& +c_{110}^{*}\left(z_{1} P_{2}+z_{2} P_{1}\right)+c_{101}^{*}\left(z_{1} P_{3}+z_{3} P_{1}\right) \\
& +c_{011}^{*}\left(z_{2} P_{3}+z_{3} P_{2}\right)
\end{aligned}
$$

By substituting (42) into the above, we obtain

$$
L(z)=\left(\begin{array}{c}
e_{11} z_{1}^{3}+e_{12} z_{2}^{3}+e_{13} z_{3}^{3}+e_{14} z_{1}^{2} z_{2}+e_{15} z_{1}^{2} z_{3}+e_{16} z_{1} z_{2}^{2}+e_{17} z_{1} z_{3}^{2}+e_{18} z_{2}^{2} z_{3}+e_{19} z_{2} z_{3}^{2}+e_{10} z_{1} z_{2} z_{3} \\
e_{21} z_{1}^{3}+e_{22} z_{2}^{3}+e_{23} z_{3}^{3}+e_{24} z_{1}^{2} z_{2}+e_{25} z_{1}^{2} z_{3}+e_{26} z_{1} z_{2}^{2}+e_{27} z_{1} z_{3}^{2}+e_{28} z_{2}^{2} z_{3}+e_{29} z_{2} z_{3}^{2}+e_{20} z_{1} z_{2} z_{3} \\
e_{31} z_{1}^{3}+e_{32} z_{2}^{3}+e_{33} z_{3}^{3}+e_{34} z_{1}^{2} z_{2}+e_{35} z_{1}^{2} z_{3}+e_{36} z_{1} z_{2}^{2}+e_{37} z_{1} z_{3}^{2}+e_{38} z_{2}^{2} z_{3}+e_{39} z_{2} z_{3}^{2}+e_{30} z_{1} z_{2} z_{3}
\end{array}\right)
$$

where

$$
\begin{aligned}
e_{11}= & \frac{a_{101}^{*} c_{200}^{*}}{2 \gamma_{1}-\gamma_{3}}+\frac{2 a_{200}^{* 2}}{\gamma_{1}}+\frac{a_{110}^{*} b_{200}^{*}}{2 \gamma_{1}-\gamma_{2}}, \\
e_{12}= & \frac{2 a_{020}^{*} b_{020}^{*}}{\gamma_{2}}+\frac{a_{020}^{*} a_{110}^{*}}{2 \gamma_{2}-\gamma_{1}}+\frac{a_{011}^{*} c_{020}^{*}}{2 \gamma_{2}-\gamma_{3}}, \\
e_{13}= & \frac{a_{002}^{*}\left(a_{101}^{*}-2 c_{002}^{*}\right)}{2 \gamma_{3}-\gamma_{1}}+\frac{a_{011}^{*} b_{002}^{*}}{2 \gamma_{3}-\gamma_{2}}, \\
e_{14}= & \frac{2 a_{020}^{*} b_{200}^{*}}{2 \gamma_{1}-\gamma_{2}}+\frac{a_{011}^{*} c_{200}^{*}}{2 \gamma_{1}-\gamma_{3}}+\frac{a_{110}^{*}\left(a_{200}^{*}+b_{110}^{*}\right)}{\gamma_{1}} \\
& +\frac{2 a_{200}^{*} a_{110}^{*}}{\gamma_{2}}, \\
e_{15}= & \frac{a_{110}^{*} b_{101}^{*}}{\gamma_{1}+\gamma_{3}-\gamma_{2}}+\frac{a_{101}^{*}\left(c_{101}^{*}-a_{200}^{*}\right)}{\gamma_{1}} \\
& +\frac{2 a_{002}^{*} c_{200}^{*}}{2 \gamma_{1}-\gamma_{3}}+\frac{a_{011}^{*} b_{200}^{*}}{2 \gamma_{1}-\gamma_{2}},
\end{aligned}
$$

$$
\begin{aligned}
e_{16}= & \frac{2 a_{020}^{*} b_{110}^{*}}{\gamma_{1}}+\frac{a_{110}^{*}\left(a_{110}^{*}+b_{020}^{*}\right)}{\gamma_{2}}+\frac{a_{101}^{*} c_{020}^{*}}{2 \gamma_{2}-\gamma_{3}} \\
& -\frac{a_{011}^{*} c_{110}^{*}}{\gamma_{2}+\gamma_{3}-\gamma_{1}}+\frac{2 a_{200}^{*} a_{020}^{*}}{2 \gamma_{2}-\gamma_{1}}, \\
e_{17}= & \frac{a_{011}^{*} b_{101}^{*}}{\gamma_{1}+\gamma_{3}-\gamma_{2}}+\frac{2 a_{200}^{*} a_{002}^{*}}{2 \gamma_{3}-\gamma_{1}}+\frac{2 a_{002}^{*} c_{101}^{*}}{\gamma_{1}}+\frac{a_{110}^{*} b_{002}^{*}}{2 \gamma_{3}-\gamma_{2}}, \\
e_{18}= & \frac{a_{110}^{*} a_{011}^{*}}{\gamma_{2}+\gamma_{3}-\gamma_{1}}+\frac{a_{020}^{*}\left(a_{101}^{*}-2 b_{011}^{*}\right)}{2 \gamma_{2}-\gamma_{1}} \\
& +\frac{a_{011}^{*}\left(b_{020}^{*}+c_{011}^{*}\right)}{\gamma_{2}}+\frac{2 a_{002}^{*} c_{020}^{*}}{2 \gamma_{2}-\gamma_{3}}, \\
e_{19}= & \frac{a_{011}^{*}\left(a_{101}^{*}-b_{011}^{*}-c_{002}^{*}\right)}{\gamma_{2}+\gamma_{3}-\gamma_{1}}+\frac{2 a_{020}^{*} b_{002}^{*}}{2 \gamma_{3}-\gamma_{2}} \\
& +\frac{2 a_{002}^{*} c_{011}^{*}}{\gamma_{2}}+\frac{a_{002}^{*} a_{110}^{*}}{2 \gamma_{3}-\gamma_{1}},
\end{aligned}
$$




$$
\begin{aligned}
& e_{10}=\frac{2 a_{200}^{*} a_{011}^{*}}{\gamma_{2}+\gamma_{3}-\gamma_{1}}+\frac{a_{101}^{*} c_{011}^{*}-a_{110}^{*} b_{011}^{*}}{\gamma_{2}}-\frac{2 a_{002}^{*} c_{110}^{*}}{2 \gamma_{3}-\gamma_{1}} \\
& +\frac{a_{011}^{*}\left(b_{110}^{*}+c_{101}^{*}\right)}{\gamma_{1}}+\frac{2 a_{020}^{*} b_{101}^{*}}{\gamma_{1}+\gamma_{3}-\gamma_{2}} ; \\
& e_{21}=\frac{2 a_{200}^{*} b_{200}^{*}}{\gamma_{1}}+\frac{b_{200}^{*} b_{110}^{*}}{2 \gamma_{1}-\gamma_{2}}+\frac{b_{101}^{*} c_{200}^{*}}{2 \gamma_{1}-\gamma_{3}} \text {, } \\
& e_{22}=\frac{a_{020}^{*} b_{110}^{*}}{2 \gamma_{2}-\gamma_{1}}+\frac{2 b_{020}^{* 2}}{\gamma_{2}}+\frac{b_{011}^{*} c_{020}^{*}}{2 \gamma_{2}-\gamma_{3}} \text {, } \\
& e_{23}=\frac{b_{002}^{*}\left(b_{011}^{*}-2 c_{002}^{*}\right)}{2 \gamma_{3}-\gamma_{2}}+\frac{a_{002}^{*} b_{101}^{*}}{2 \gamma_{3}-\gamma_{1}}, \\
& e_{24}=\frac{2 b_{020}^{*} b_{200}^{*}}{2 \gamma_{1}-\gamma_{2}}+\frac{b_{011}^{*} c_{200}^{*}}{2 \gamma_{1}-\gamma_{3}} \\
& +\frac{b_{110}^{*}\left(a_{200}^{*}+b_{110}^{*}\right)}{\gamma_{1}}+\frac{2 b_{200}^{*} a_{110}^{*}}{\gamma_{2}}, \\
& e_{25}=\frac{b_{110}^{*} b_{101}^{*}}{\gamma_{1}+\gamma_{3}-\gamma_{2}}+\frac{b_{101}^{*}\left(c_{101}^{*}+a_{200}^{*}\right)}{\gamma_{1}} \\
& +\frac{2 b_{002}^{*} c_{200}^{*}}{2 \gamma_{1}-\gamma_{3}}+\frac{b_{200}^{*}\left(b_{011}^{*}-2 a_{101}^{*}\right)}{2 \gamma_{1}-\gamma_{2}}, \\
& e_{26}=\frac{2 b_{020}^{*} b_{110}^{*}}{\gamma_{1}}+\frac{b_{110}^{*}\left(a_{110}^{*}+b_{020}^{*}\right)}{\gamma_{2}} \\
& +\frac{b_{101}^{*} c_{020}^{*}}{2 \gamma_{2}-\gamma_{3}}+\frac{2 b_{200}^{*} a_{020}^{*}}{2 \gamma_{2}-\gamma_{1}} \\
& e_{27}=\frac{b_{101}^{*}\left(b_{011}^{*}-a_{101}^{*}-c_{002}^{*}\right)}{\gamma_{1}+\gamma_{3}-\gamma_{2}}+\frac{2 b_{200}^{*} a_{002}^{*}}{2 \gamma_{3}-\gamma_{1}} \\
& +\frac{2 b_{002}^{*} c_{101}^{*}}{\gamma_{1}}+\frac{b_{110}^{*} b_{002}^{*}}{2 \gamma_{3}-\gamma_{2}} \\
& e_{28}=\frac{b_{110}^{*} a_{011}^{*}}{\gamma_{2}+\gamma_{3}-\gamma_{1}}+\frac{a_{020}^{*} b_{101}^{*}}{2 \gamma_{2}-\gamma_{1}} \\
& +\frac{b_{011}^{*}\left(c_{011}^{*}-b_{020}^{*}\right)}{\gamma_{2}}+\frac{2 b_{002}^{*} c_{020}^{*}}{2 \gamma_{2}-\gamma_{3}}, \\
& e_{29}=\frac{a_{011}^{*} b_{101}^{*}}{\gamma_{2}+\gamma_{3}-\gamma_{1}}+\frac{2 b_{020}^{*} b_{002}^{*}}{2 \gamma_{3}-\gamma_{2}}+\frac{2 b_{002}^{*} c_{011}^{*}}{\gamma_{2}}+\frac{a_{002}^{*} b_{110}^{*}}{2 \gamma_{3}-\gamma_{1}} \text {, } \\
& e_{20}=\frac{2 b_{200}^{*} a_{011}^{*}}{\gamma_{2}+\gamma_{3}-\gamma_{1}}+\frac{b_{101}^{*}\left(c_{011}^{*}+a_{110}^{*}\right)}{\gamma_{2}}-\frac{2 b_{002}^{*} c_{110}^{*}}{2 \gamma_{3}-\gamma_{2}} \\
& +\frac{b_{011}^{*} c_{101}^{*}-b_{110}^{*} a_{101}^{*}}{\gamma_{1}}+\frac{2 b_{020}^{*} b_{101}^{*}}{\gamma_{1}+\gamma_{3}-\gamma_{2}} ; \\
& e_{31}=\frac{c_{101}^{*} c_{200}^{*}}{2 \gamma_{1}-\gamma_{3}}+\frac{2 a_{200}^{*} c_{200}^{*}}{\gamma_{1}}+\frac{c_{110}^{*} b_{200}^{*}}{2 \gamma_{1}-\gamma_{2}} \text {, } \\
& e_{32}=\frac{2 c_{020}^{*} b_{020}^{*}}{\gamma_{2}}+\frac{a_{020}^{*} c_{110}^{*}}{2 \gamma_{2}-\gamma_{1}}+\frac{c_{011}^{*} c_{020}^{*}}{2 \gamma_{2}-\gamma_{3}},
\end{aligned}
$$

$$
\begin{aligned}
& e_{33}=\frac{a_{002}^{*} c_{101}^{*}}{2 \gamma_{3}-\gamma_{1}}+\frac{c_{011}^{*} b_{002}^{*}}{2 \gamma_{3}-\gamma_{2}}, \\
& e_{34}=\frac{2 c_{020}^{*} b_{200}^{*}}{2 \gamma_{1}-\gamma_{2}}+\frac{c_{011}^{*} c_{200}^{*}}{2 \gamma_{1}-\gamma_{3}} \\
& +\frac{c_{110}^{*}\left(a_{200}^{*}+b_{110}^{*}-c_{101}^{*}\right)}{\gamma_{1}}+\frac{2 c_{200}^{*} a_{110}^{*}}{\gamma_{2}}, \\
& e_{35}=\frac{c_{110}^{*} b_{101}^{*}}{\gamma_{1}+\gamma_{3}-\gamma_{2}}+\frac{c_{101}^{*}\left(c_{101}^{*}+a_{200}^{*}\right)}{\gamma_{1}} \\
& +\frac{2 c_{200}^{*}\left(c_{002}^{*}-a_{101}^{*}\right)}{2 \gamma_{1}-\gamma_{3}}+\frac{c_{011}^{*} b_{200}^{*}}{2 \gamma_{1}-\gamma_{2}}, \\
& e_{36}=\frac{2 c_{020}^{*} b_{110}^{*}}{\gamma_{1}}+\frac{c_{110}^{*}\left(a_{110}^{*}+b_{020}^{*}-c_{011}^{*}\right)}{\gamma_{2}} \\
& +\frac{c_{101}^{*} c_{020}^{*}}{2 \gamma_{2}-\gamma_{3}}+\frac{2 c_{200}^{*} a_{020}^{*}}{2 \gamma_{2}-\gamma_{1}}, \\
& e_{37}=\frac{c_{011}^{*} b_{101}^{*}}{\gamma_{1}+\gamma_{3}-\gamma_{2}}+\frac{2 c_{200}^{*} a_{002}^{*}}{2 \gamma_{3}-\gamma_{1}} \\
& +\frac{c_{101}^{*}\left(c_{002}^{*}-a_{101}^{*}\right)}{\gamma_{1}}+\frac{c_{110}^{*} b_{002}^{*}}{2 \gamma_{3}-\gamma_{2}} \\
& e_{38}=\frac{c_{110}^{*} a_{011}^{*}}{\gamma_{2}+\gamma_{3}-\gamma_{1}}+\frac{a_{020}^{*} c_{101}^{*}}{2 \gamma_{2}-\gamma_{1}}+\frac{c_{011}^{*}\left(b_{020}^{*}+c_{011}^{*}\right)}{\gamma_{2}} \\
& +\frac{2 c_{020}^{*}\left(c_{002}^{*}-b_{011}^{*}\right)}{2 \gamma_{2}-\gamma_{3}} \\
& e_{39}=\frac{a_{011}^{*} c_{101}^{*}}{\gamma_{2}+\gamma_{3}-\gamma_{1}}+\frac{2 c_{020}^{*} b_{002}^{*}}{2 \gamma_{3}-\gamma_{2}} \\
& +\frac{c_{011}^{*}\left(c_{002}^{*}-b_{011}^{*}\right)}{\gamma_{2}}+\frac{a_{002}^{*} c_{110}^{*}}{2 \gamma_{3}-\gamma_{1}}, \\
& e_{30}=\frac{2 c_{200}^{*} a_{011}^{*}}{\gamma_{2}+\gamma_{3}-\gamma_{1}}+\frac{c_{011}^{*}\left(b_{110}^{*}+c_{101}^{*}\right)}{\gamma_{2}} \\
& +\frac{c_{011}^{*}\left(b_{110}^{*}+c_{101}^{*}\right)}{\gamma_{1}}+\frac{2 c_{020}^{*} b_{101}^{*}}{\gamma_{1}+\gamma_{3}-\gamma_{2}} \text {. }
\end{aligned}
$$

We make a further change $z=w+Q(w) \equiv h_{2}(w)$, where $Q=\left(Q_{1}, Q_{2}, Q_{3}\right)$ is homogeneous cubic polynomial, so that (48) becomes

$$
\begin{aligned}
\dot{w} & =\left[D h_{2}(w)\right]^{-1} \cdot \dot{z} \\
& =\left(I-D Q+O\left(|D Q|^{2}\right)\right) \cdot \dot{z}
\end{aligned}
$$




$$
\begin{gathered}
=\left(\begin{array}{c}
\gamma_{1} w_{1}+a_{101}^{*} w_{1} w_{3} \\
\gamma_{2} w_{2}+b_{011}^{*} w_{2} w_{3} \\
\gamma_{3} w_{3}+c_{002}^{*} w_{3}^{2}+c_{110}^{*} w_{1} w_{2}
\end{array}\right) \\
+\left(\begin{array}{c}
\gamma_{1} Q_{1}-\sum_{j=1}^{3} Q_{1 j} \gamma_{j} w_{j} \\
\gamma_{2} Q_{2}-\sum_{j=1}^{3} Q_{2 j} \gamma_{j} w_{j} \\
\gamma_{3} Q_{3}-\sum_{j=1}^{3} Q_{3 j} \gamma_{j} w_{j}
\end{array}\right) \\
+L(w)+O\left(w^{4}\right)
\end{gathered}
$$

where $Q_{i j}=\partial Q_{i} / \partial w_{j}, i, j=1,2,3$ and $L$ has the form as before. In order to eliminate some possibly cubic terms, we consider the equations below

$$
\gamma_{i} Q_{i}-\sum_{j=1}^{3} Q_{i j} \gamma_{j} w_{j}+L_{i}(w)=0, \quad i=1,2,3
$$

Suppose that for $i=1,2,3$,

$$
\begin{aligned}
Q_{i}= & q_{i 1} w_{1}^{3}+q_{i 2} w_{2}^{3}+q_{i 3} w_{3}^{3}+q_{i 4} w_{1}^{2} w_{2}+q_{i 5} w_{1}^{2} w_{3}+q_{i 6} w_{1} w_{2}^{2} \\
& +q_{i 7} w_{1} w_{3}^{2}+q_{i 8} w_{2}^{2} w_{3}+q_{i 9} w_{2} w_{3}^{2}+q_{i 0} w_{1} w_{2} w_{3} .
\end{aligned}
$$

By inserting these representations into (54), we can solve as before

$$
\begin{aligned}
Q_{1}= & \frac{e_{11}}{2 \gamma_{1}} w_{1}^{3}+\frac{e_{12}}{3 \gamma_{2}-\gamma_{1}} w_{2}^{3}+\frac{e_{13}}{3 \gamma_{3}-\gamma_{1}} w_{3}^{3} \\
& +\frac{e_{15}}{\gamma_{1}+\gamma_{3}} w_{1}^{2} w_{3}+\frac{e_{16}}{2 \gamma_{2}} w_{1} w_{2}^{2} \\
& +\frac{e_{18}}{2 \gamma_{2}+\gamma_{3}-\gamma_{1}} w_{2}^{2} w_{3} \\
& +\frac{e_{19}}{\gamma_{2}+2 \gamma_{3}-\gamma_{1}} w_{2} w_{3}^{2} \\
& +\frac{e_{10}}{\gamma_{2}+\gamma_{3}} w_{1} w_{2} w_{3}, \\
Q_{2}= & \frac{e_{21}}{3 \gamma_{1}-\gamma_{2}} w_{1}^{3}+\frac{e_{22}}{2 \gamma_{2}} w_{2}^{3}+\frac{e_{23}}{3 \gamma_{3}-\gamma_{2}} w_{3}^{3} \\
& +\frac{e_{24}}{2 \gamma_{1}} w_{1}^{2} w_{2}+\frac{e_{25}}{2 \gamma_{1}+\gamma_{3}-\gamma_{2}} w_{1}^{2} w_{3} \\
& +\frac{e_{27}}{\gamma_{1}+2 \gamma_{3}-\gamma_{2}} w_{1} w_{3}^{2}+\frac{e_{28}}{\gamma_{2}+\gamma_{3}} w_{2}^{2} w_{3} \\
& +\frac{e_{20}}{\gamma_{1}+\gamma_{3}} w_{1} w_{2} w_{3},
\end{aligned}
$$

$$
\begin{aligned}
Q_{3}= & \frac{e_{31}}{3 \gamma_{1}-\gamma_{3}} w_{1}^{3}+\frac{e_{32}}{3 \gamma_{2}-\gamma_{3}} w_{2}^{3} \\
& +\frac{e_{34}}{2 \gamma_{1}+\gamma_{2}-\gamma_{3}} w_{1}^{2} w_{2}+\frac{e_{35}}{2 \gamma_{1}} w_{1}^{2} w_{3} \\
& +\frac{e_{36}}{\gamma_{1}+2 \gamma_{2}-\gamma_{3}} w_{1} w_{2}^{2}+\frac{e_{37}}{\gamma_{1}+\gamma_{3}} w_{1} w_{3}^{2} \\
& +\frac{e_{38}}{2 \gamma_{2}} w_{2}^{2} w_{3}+\frac{e_{39}}{\gamma_{2}+\gamma_{3}} w_{2} w_{3}^{2} .
\end{aligned}
$$

Hence, system (53) becomes now

$$
\begin{aligned}
\dot{w}_{1}= & \gamma_{1} w_{1}+a_{101}^{*} w_{1} w_{3}+e_{14} w_{1}^{2} w_{2}+e_{17} w_{1} w_{3}^{2} \\
& +O\left(\left|w_{1}, w_{2}, w_{3}\right|^{4}\right), \\
\dot{w}_{2}= & \gamma_{2} w_{2}+b_{011}^{*} w_{2} w_{3}+e_{26} w_{1} w_{2}^{2}+e_{29} w_{2} w_{3}^{2} \\
& +O\left(\left|w_{1}, w_{2}, w_{3}\right|^{4}\right), \\
\dot{w}_{3}= & \gamma_{3} w_{3}+c_{002}^{*} w_{3}^{2}+c_{110}^{*} w_{1} w_{2}+e_{33} w_{3}^{3}+e_{30} w_{1} w_{2} w_{3} \\
& +O\left(\left|w_{1}, w_{2}, w_{3}\right|^{4}\right),
\end{aligned}
$$

where $w$ and all of the coefficients are complex. Finally making the change $w=T x$ and then taking the real parts of $x$ and the coefficients of all terms of the resulting system, we can get a cubic real normal form of the form (7) with

$$
\begin{aligned}
& a_{1}=\frac{1}{4}\left(2 \tilde{a}_{101}+\tilde{b}_{011}-\tilde{a}_{011}\right), \\
& b_{1}=\frac{1}{4}\left(\tilde{a}_{011}+\tilde{b}_{011}-2 \tilde{b}_{101}\right) \text {, } \\
& c_{1}=\frac{1}{2}\left(\widetilde{c}_{200}+\widetilde{c}_{020}\right) \text {, } \\
& d_{1}=\widetilde{c}_{002} \text {, } \\
& a_{2}=\frac{1}{8\left(\lambda_{1}^{2}+1\right)}\left[\lambda _ { 1 } \left(5 \tilde{a}_{200}^{2}+5 \tilde{b}_{020}^{2}-\tilde{a}_{020}^{2}-\tilde{b}_{200}^{2}\right.\right. \\
& +4 \widetilde{a}_{200} \widetilde{a}_{020}+4 \widetilde{b}_{200} \widetilde{b}_{020}+3 \widetilde{b}_{110} \\
& \left.\times\left(\tilde{a}_{200}+\tilde{a}_{020}\right)+3 \tilde{a}_{110}\left(\tilde{b}_{200}+\tilde{b}_{020}\right)\right) \\
& -2 \widetilde{a}_{200} \widetilde{b}_{200}+2 \widetilde{a}_{020} \widetilde{b}_{020} \\
& \left.+\widetilde{a}_{110}\left(\widetilde{a}_{200}+\widetilde{a}_{020}\right)-\widetilde{b}_{110}\left(\widetilde{b}_{200}+\widetilde{b}_{020}\right)\right] \\
& -\frac{\lambda_{1}}{8\left(\lambda_{1}^{2}+9\right)} \\
& \times\left[\left(\widetilde{a}_{020}-\widetilde{a}_{200}+\widetilde{b}_{110}\right)^{2}+\left(-\widetilde{a}_{110}+\widetilde{b}_{020}-\widetilde{b}_{200}\right)^{2}\right]
\end{aligned}
$$




$$
\begin{aligned}
& -\frac{1}{8\left[\left(2 \lambda_{1}-\lambda_{2}\right)^{2}+4\right]} \\
& \times\left(-2\left(\left(\tilde{a}_{011}+\tilde{b}_{101}\right)\left(\widetilde{c}_{200}-\widetilde{c}_{020}\right)\right.\right. \\
& \left.+\left(-\widetilde{a}_{101}+\widetilde{b}_{011}\right) \widetilde{c}_{110}\right) \\
& +\left(2 \lambda_{1}-\lambda_{2}\right)\left(\left(-\widetilde{a}_{101}+\widetilde{b}_{011}\right)\left(\widetilde{c}_{200}-\widetilde{c}_{020}\right)\right. \\
& \left.\left.-\left(\tilde{a}_{011}+\tilde{b}_{101}\right) \widetilde{c}_{110}\right)\right) \\
& b_{2}=\frac{1}{8\left(\lambda_{1}^{2}+1\right)} \\
& \times\left(-\lambda_{1}\left(6 \widetilde{a}_{200} \widetilde{b}_{200}-6 \widetilde{a}_{020} \widetilde{b}_{020}+3 \widetilde{b}_{110}\left(\widetilde{b}_{200}+\widetilde{b}_{020}\right)\right.\right. \\
& \left.-3 \widetilde{a}_{110}\left(\widetilde{a}_{200}+\widetilde{a}_{020}\right)\right)+\widetilde{a}_{200}^{2}+3 \widetilde{a}_{020}^{2}+3 \widetilde{b}_{200}^{2} \\
& +\widetilde{b}_{020}^{2}+4 \widetilde{a}_{200} \widetilde{a}_{020}+4 \widetilde{b}_{200} \widetilde{b}_{020}-\widetilde{b}_{110}\left(\widetilde{a}_{200}+\widetilde{a}_{020}\right) \\
& \left.-\widetilde{a}_{110}\left(\widetilde{b}_{200}+\widetilde{b}_{020}\right)\right) \frac{3}{8\left(\lambda_{1}^{2}+9\right)} \\
& \times\left(\left(\widetilde{a}_{020}-\widetilde{a}_{200}+\widetilde{b}_{110}\right)^{2}+\left(-\widetilde{a}_{110}+\widetilde{b}_{020}-\widetilde{b}_{200}\right)^{2}\right) \\
& -\frac{1}{8\left[\left(2 \lambda_{1}-\lambda_{2}\right)^{2}+4\right]} \\
& \times\left(( 2 \lambda _ { 1 } - \lambda _ { 2 } ) \left(\left(\tilde{a}_{011}+\tilde{b}_{101}\right)\left(\widetilde{c}_{200}-\widetilde{c}_{020}\right)\right.\right. \\
& \left.+\left(-\tilde{a}_{101}+\tilde{b}_{011}\right) \tilde{c}_{110}\right) \\
& +2\left(\left(-\widetilde{a}_{101}+\tilde{b}_{011}\right)\left(\widetilde{c}_{200}-\widetilde{c}_{020}\right)\right. \\
& \left.\left.-\left(\widetilde{a}_{011}+\widetilde{b}_{101}\right) \widetilde{c}_{110}\right)\right) \\
& a_{3}=\frac{1}{2\left[\left(2 \lambda_{2}-\lambda_{1}\right)^{2}+1\right]} \\
& \times\left(\left(2 \lambda_{2}-\lambda_{1}\right)\right. \\
& \times\left(\widetilde{a}_{002}\left(2 \tilde{a}_{200}+\widetilde{b}_{110}\right)+\widetilde{b}_{002}\left(2 \tilde{b}_{020}+\tilde{a}_{110}\right)\right) \\
& \left.+\widetilde{a}_{002}\left(2 \widetilde{b}_{020}+\widetilde{a}_{110}\right)-\widetilde{b}_{002}\left(2 \widetilde{a}_{200}+\widetilde{b}_{110}\right)\right) \\
& +\widetilde{c}_{011}\left(\lambda _ { 2 } \left(\left(\widetilde{a}_{011}+\widetilde{b}_{101}\right)\left(\widetilde{b}_{101}-\widetilde{b}_{011}\right)-\left(-\widetilde{a}_{101}+\widetilde{b}_{011}\right)\right.\right. \\
& \left.\times\left(\tilde{a}_{101}-\tilde{a}_{011}\right)\right) \\
& +2\left(\left(\widetilde{a}_{011}+\widetilde{b}_{101}\right)\left(\widetilde{a}_{101}-\widetilde{a}_{011}\right)\right. \\
& \left.\left.+\left(-\widetilde{a}_{101}+\widetilde{b}_{011}\right)\left(\widetilde{b}_{101}-\widetilde{b}_{011}\right)\right)\right) \\
& +\frac{1}{\lambda_{1}^{2}+1}\left(\lambda_{1} \widetilde{a}_{002}+\tilde{b}_{002}\right)\left(\widetilde{a}_{101}-\widetilde{a}_{011}\right) \text {, }
\end{aligned}
$$

$$
\begin{aligned}
& b_{3}=\frac{1}{2\left[\left(2 \lambda_{2}-\lambda_{1}\right)^{2}+1\right]} \\
& \times\left(\left(\tilde{a}_{002}\left(2 \widetilde{a}_{020}-\widetilde{b}_{110}\right)+\widetilde{b}_{002}\left(2 \widetilde{b}_{200}-\widetilde{a}_{110}\right)\right)\right. \\
& -\left(2 \lambda_{2}-\lambda_{1}\right) \\
& \left.\times\left(\tilde{a}_{002}\left(2 \tilde{b}_{200}-\tilde{a}_{110}\right)-\tilde{b}_{002}\left(2 \tilde{a}_{020}-\tilde{b}_{110}\right)\right)\right) \\
& +\frac{1}{4\left(\lambda_{2}^{2}+4\right)}\left(2 \left(\left(\tilde{a}_{011}+\tilde{b}_{101}\right)\left(\tilde{b}_{101}-\tilde{b}_{011}\right)\right.\right. \\
& \left.-\left(-\tilde{a}_{101}+\tilde{b}_{011}\right)\left(\tilde{a}_{101}-\tilde{a}_{011}\right)\right) \\
& -\lambda_{2}\left(\left(\widetilde{a}_{011}+\widetilde{b}_{101}\right)\left(\widetilde{a}_{101}-\widetilde{a}_{011}\right)\right. \\
& +\left(-\widetilde{a}_{101}+\tilde{b}_{011}\right) \\
& \left.\left.\times\left(\widetilde{b}_{101}-\widetilde{b}_{011}\right)\right)\right)+\frac{1}{\lambda_{1}^{2}+1} \\
& \times\left(\tilde{a}_{002}-\lambda_{1} \widetilde{b}_{002}\right)\left(\tilde{a}_{101}-\tilde{a}_{011}\right), \\
& c_{2}=\frac{\lambda_{1}}{2\left(\lambda_{1}^{2}+1\right)}\left[\widetilde{c}_{011}\left(\widetilde{b}_{200}+\widetilde{b}_{020}\right)\right. \\
& \left.+\widetilde{c}_{101}\left(\widetilde{a}_{200}+\widetilde{a}_{020}+\widetilde{c}_{101}-\widetilde{c}_{011}\right)\right] \\
& +\frac{\lambda_{2}}{4\left(\lambda_{2}^{2}+4\right)}\left(\widetilde{c}_{110}\left(\tilde{a}_{011}+2 \tilde{b}_{101}-\tilde{b}_{011}\right)\right. \\
& +\left(2 \widetilde{a}_{101}-\widetilde{b}_{011}-\widetilde{a}_{011}\right) \\
& \left.\times\left(\widetilde{c}_{200}-\widetilde{c}_{020}\right)\right) \\
& +\frac{1}{2\left(\lambda_{2}^{2}+4\right)}\left(\widetilde{c}_{110}\left(2 \widetilde{a}_{101}-\widetilde{b}_{011}-\widetilde{a}_{011}\right)\right. \\
& -\left(\tilde{a}_{011}+2 \tilde{b}_{101}-\tilde{b}_{011}\right) \\
& \left.\times\left(\widetilde{c}_{200}-\widetilde{c}_{020}\right)\right), \\
& d_{2}=\frac{2 \lambda_{2}-\lambda_{1}}{2\left[\left(2 \lambda_{2}-\lambda_{1}\right)^{2}+1\right]} \\
& \times\left(2 \widetilde{a}_{002} \widetilde{c}_{101}-\widetilde{a}_{002} \widetilde{c}_{011}+\widetilde{b}_{002} \widetilde{c}_{011}\right) \\
& +\frac{1}{2\left[\left(2 \lambda_{2}-\lambda_{1}\right)^{2}+1\right]} \\
& \times\left(\widetilde{a}_{002} \widetilde{\mathcal{c}}_{011}-2 \widetilde{b}_{002} \widetilde{\mathcal{c}}_{101}+\widetilde{b}_{002} \widetilde{\mathcal{c}}_{011}\right) .
\end{aligned}
$$

Then, by the equations in (36), we finally get the relationship between the coefficients of the system (2) and of the normal form (7). 


\section{Examples}

4.1. An Example about the Existence of a Limit Cycle in Three-Dimensional Lotka-Volterra Systems. In this section, we construct a concrete example of three-dimensional LotkaVolterra systems according to Theorem 1 . It is shown that this system undergoes nonisolated zero-Hopf bifurcation.

We consider the following three-parameter LotkaVolterra system in the first octant $\mathbb{R}_{+}^{3}$. Consider

$$
\begin{aligned}
& \frac{d x}{d t}=x(-v x+v y+v z-v) \\
& \frac{d y}{d t}=y(-2 v x-2 v y-v z+5 v) \\
& \frac{d z}{d t}=\frac{z}{2 v^{2}}\left(-x\left(6 v^{3}+6 v^{2} \varepsilon+10 v^{2} u \varepsilon\right.\right. \\
& \left.+6 v u \varepsilon^{2}+3 v u^{2} \varepsilon^{2}+u^{2} \varepsilon^{3}\right) \\
& -y\left(-6 v^{3}-2 v^{2} u \varepsilon+2 v u \varepsilon^{2}+v u^{2} \varepsilon^{2}+u^{2} \varepsilon^{3}\right) \\
& +z\left(6 v^{3}+2 v^{2} \varepsilon+4 v^{2} u \varepsilon\right) \\
& +\left(-6 v^{3}+4 v^{2} \varepsilon+4 v^{2} u \varepsilon+8 v u \varepsilon^{2}\right. \\
& \left.\left.+4 v u^{2} \varepsilon^{2}+2 u^{2} \varepsilon^{3}\right)\right)
\end{aligned}
$$

where $0<\varepsilon \ll 1, v>0$ and $u$ are bounded parameters.

First of all, we need to change the system (59) to the form of system (2) as in [14]. It can be checked that the point $(1,1,1)$ is zero-Hopf equilibrium of system (59). We do the change of variables $X=x-1, Y=y-1$, and $Z=z-1$ to obtain

$$
\begin{aligned}
\frac{d X}{d t}=v(1+X)(-X+Y+Z) & \\
\frac{d Y}{d t}=v(1+Y)(-2 X-2 Y-Z) & \\
\frac{d Z}{d t}=\frac{1+Z}{2 v^{2}}( & -X\left(6 v^{3}+6 v^{2} \varepsilon+10 v^{2} u \varepsilon+6 v u \varepsilon^{2}\right. \\
& \left.+3 v u^{2} \varepsilon^{2}+u^{2} \varepsilon^{3}\right) \\
& -Y\left(-6 v^{3}-2 v^{2} u \varepsilon+2 v u \varepsilon^{2}+v u^{2} \varepsilon^{2}+u^{2} \varepsilon^{3}\right) \\
& \left.+Z\left(6 v^{3}+2 v^{2} \varepsilon+4 v^{2} u \varepsilon\right)\right) .
\end{aligned}
$$

The Jacobian matrix of system $(60)$ at $(0,0,0)$ has eigenvalues $\varepsilon, \varepsilon u+v i$ and $\varepsilon u-v i$ with $v>0$. According to [14], in order to obtain the real Jordan normal form of system (60) at the origin, we do the linear transformation

$$
\left(\begin{array}{c}
U_{1} \\
V_{1} \\
W_{1}
\end{array}\right)=\left(\begin{array}{ccc}
p_{11} & p_{12} & p_{13} \\
p_{21} & 1 & 0 \\
p_{31} & p_{32} & 1
\end{array}\right)\left(\begin{array}{l}
X \\
Y \\
Z
\end{array}\right)
$$

where $p_{11}=-\left(u \varepsilon^{2}+3 v \varepsilon+3 v u \varepsilon+5 v^{2}\right) /(v(v+\varepsilon)), p_{12}=-\left(-v^{2}+\right.$ $\left.v u \varepsilon+v \varepsilon+u \varepsilon^{2}\right) /(v(v+\varepsilon)), p_{13}=2 v /(v+\varepsilon), p_{21}=(3 v+$ $\varepsilon) /(v+\varepsilon), p_{31}=-\left(6 v^{2}+6 v u \varepsilon+u^{2} \varepsilon^{2}\right) / 2 v^{2}$, and $p_{32}=-u \varepsilon(2 v+$ $u \varepsilon) / 2 v^{2}$. Then, in the new variables $\left(U_{1}, V_{1}\right.$, and $\left.W_{1}\right)$ system (60) becomes

$$
\begin{aligned}
& \frac{d U_{1}}{d t}=u \varepsilon U_{1}+v V_{1}+\sum_{i+j+k=2} a_{i j k} U_{1}^{i} V_{1}^{j} W_{1}^{k}, \\
& \frac{d V_{1}}{d t}=-v U_{1}+u \varepsilon V_{1}+\sum_{i+j+k=2} b_{i j k} U_{1}^{i} V_{1}^{j} W_{1}^{k}, \\
& \frac{d W_{1}}{d t}=\varepsilon W_{1}+\sum_{i+j+k=2} c_{i j k} U_{1}^{i} V_{1}^{j} W_{1}^{k},
\end{aligned}
$$

where $a_{i j k}, b_{i j k}$, and $c_{i j k}$ have the following expressions:

$$
\begin{aligned}
& a_{011}=21 v+(-11+90 u) \varepsilon+O\left(\varepsilon^{2}\right), \\
& a_{020}=3 v+(24-17 u) \varepsilon+O\left(\varepsilon^{2}\right) \\
& a_{002}=-18 v-(36 u+30) \varepsilon+O\left(\varepsilon^{2}\right) \\
& a_{110}=29 v+\left(\frac{16}{3}+93 u\right) \varepsilon+O\left(\varepsilon^{2}\right) \\
& a_{101}=-30 v-(60 u+72) \varepsilon+O\left(\varepsilon^{2}\right), \\
& a_{200}=-9 v-(22 u+40) \varepsilon+O\left(\varepsilon^{2}\right) \\
& b_{101}=-6 v+22 \varepsilon+O\left(\varepsilon^{2}\right) \\
& c_{020}=(36-32 u) \varepsilon+O\left(\varepsilon^{2}\right) \\
& c_{011}=-12 v-(6 u+15) \varepsilon+O\left(\varepsilon^{2}\right) \\
& c_{011}=30 v+(10+105 u) \varepsilon+O\left(\varepsilon^{2}\right) \\
& b_{110}=-9 v-24 \varepsilon+O\left(\varepsilon^{2}\right) \\
& c_{002}=12 \varepsilon+O\left(\varepsilon^{2}\right) \\
& b_{200}=-6 v+8 \varepsilon+O\left(\varepsilon^{2}\right) \\
& b_{020}=4 v+\left(-\frac{22}{3}+15 u\right) \varepsilon+O\left(\varepsilon^{2}\right) \\
& b_{110}=-27 v-(123+54 u) \varepsilon+O\left(\varepsilon^{2}\right),
\end{aligned}
$$


Next, we need to calculate the partial coefficients of the normal form of system (63). We can get $\widetilde{a}_{i j k}, \widetilde{b}_{i j k}$, and $\widetilde{c}_{i j k}$ by (36), and then by the formulas of (58) we have

$$
\begin{gathered}
a_{1}=\frac{21}{4}+\frac{1}{v}(12+24 u) \varepsilon+O\left(\varepsilon^{2}\right), \\
a_{2}=90-\frac{1}{16 v}(5875+6244 u) \varepsilon+O\left(\varepsilon^{2}\right), \\
a_{3}=45-97740 v \\
-\frac{3}{v}(121-264 u+31860 v+299790 u v) \varepsilon+O\left(\varepsilon^{2}\right), \\
c_{1}=-3-\frac{12}{v}(1+2 u) \varepsilon+O\left(\varepsilon^{2}\right) \\
c_{2}=-\frac{657}{4}+\frac{3}{8 v}(-3263+226 u) \varepsilon+O\left(\varepsilon^{2}\right) \\
d_{1}=-18-\frac{3}{v}(19+12 u) \varepsilon+O\left(\varepsilon^{2}\right) \\
d_{2}=-756-\frac{9}{v}(436+351 u) \varepsilon+O\left(\varepsilon^{2}\right) .
\end{gathered}
$$

By Theorem 1, we have the following conclusion.

Theorem 3. For any given $\varepsilon_{0}>0$, suppose that $-7 /\left(24+7 \varepsilon_{0}\right)<$ $u<0$, and then for $0<\varepsilon \ll \varepsilon_{0}$, (59) has a periodic orbit near the origin, which is unstable.

Proof. In this example, it is easy to see that $\delta_{1}=1, \delta_{2}=1 / u$. From (64) and (10) we can get $\widetilde{c}_{1}=\widetilde{d}_{1}<0$, thus, in order to satisfy (18), we need

$$
\widetilde{d}_{1}-\delta_{1} \delta_{2}=-\frac{24}{7}-\frac{1}{u}+\frac{4}{49} \frac{-37+108 u}{v} \varepsilon+O\left(\varepsilon^{2}\right)>0 .
$$

For any given $\varepsilon_{0}>0$, suppose that $-7 /\left(24+7 \varepsilon_{0}\right)<u<0$. It can be checked that $\widetilde{d}_{1}-\delta_{1} \delta_{2}>0$ for $0<\varepsilon \ll \varepsilon_{0}$. Then, by Theorem 1, (59) has a periodic orbit near the origin. Next, we consider the stability of the periodic orbit.

From (64), we can also get

$$
\begin{aligned}
\Delta= & \frac{49+280 u+960 u^{2}}{49 u^{2}}-\frac{16}{343} \\
& \times \frac{-259-502 u+3672 u^{2}}{u v}+O\left(\varepsilon^{2}\right)>0,
\end{aligned}
$$

when $-7 /\left(24+7 \varepsilon_{0}\right)<u<0$ holds, where $\Delta$ is given by (20). So none of the conditions (a), (b), or (c) in Theorem 1 holds; further, we know that the periodic orbit is unstable.

Remark 4. From (63), we can find out that system (59) does not satisfy the conditions mentioned in [14]. Thus, we cannot use the results in [14] to study the existence of a limit cycle in (59).
4.2. An Example about the Existence of an Invariant Torus. For convenience, we give an example about the existence of an invariant torus in a system, which has the form of (2). We consider the following system in the first octant $\mathbb{R}_{+}^{3}$ :

$$
\begin{aligned}
\frac{d U_{2}}{d t}= & -\frac{5}{24} \varepsilon U_{2}-\frac{5}{24} V_{2}+3 U_{2}^{2}-2 V_{2}^{2} \\
& +3 W_{2}^{2}-15 U_{2} V_{2}+2 U_{2} W_{2}+5 V_{2} W_{2}, \\
\frac{d V_{2}}{d t}= & \frac{5}{24} U_{2}-\frac{5}{24} \varepsilon V_{2}+9 U_{2}^{2}-5 V_{2}^{2}+6 W_{2}^{2} \\
& +U_{2} V_{2}-4 V_{2} W_{2} \\
\frac{d W_{2}}{d t}= & \varepsilon W_{2}-4 U_{2}^{2}+8 V_{2}^{2}+3 W_{2}^{2}+510 U_{2} V_{2} \\
& -7 U_{2} W_{2}-5 V_{2} W_{2},
\end{aligned}
$$

where $0<\varepsilon \ll 1$.

According to Section 3, we have

$$
\begin{gathered}
a_{1}=6, \\
a_{2}=-\frac{116136}{25}+\frac{1409296}{125} \varepsilon+O\left(\varepsilon^{2}\right), \\
a_{3}=\frac{180576}{25}+\frac{2081376}{125} \varepsilon+O\left(\varepsilon^{2}\right), \\
c_{1}=-\frac{48}{5}, \\
c_{2}=\frac{117936}{25}-\frac{3953376}{125} \varepsilon+O\left(\varepsilon^{2}\right), \\
d_{1}=-\frac{72}{5} \\
d_{2}=\frac{11232}{25}+\frac{870048}{125} \varepsilon+O\left(\varepsilon^{2}\right) .
\end{gathered}
$$

Let $A_{1}=1666324684 / 40625$ and $A_{2}=1731664 / 3125$. Then, we have the following theorem.

Theorem 5. For any given $0<\varepsilon_{1}<A_{1}-A_{2}$, there exists an $\varepsilon_{0}>0$ such that for $0<\varepsilon<\varepsilon_{0}$, (68) has a unique invariant torus near the origin, which is unstable.

Proof. By (69) and (29), we can obtain

$$
\begin{aligned}
& \delta_{0}=\frac{14892}{125}-\frac{1753792}{375} \varepsilon-\frac{92710806}{3125} \varepsilon^{2}+O\left(\varepsilon^{3}\right), \\
& \Delta_{0}=\frac{13251}{50}+\frac{216458}{1875} \varepsilon+\frac{3772653}{12500} \varepsilon^{2}+O\left(\varepsilon^{3}\right), \\
& \delta_{0}^{\prime}=-\frac{248104}{1625}-\frac{70129552}{14625} \varepsilon-\frac{1217815988}{40625} \varepsilon^{2}+O\left(\varepsilon^{2}\right) .
\end{aligned}
$$

Thus, for $0<\varepsilon \ll 1, \Delta_{0}>0$. Further, we can get

$$
\Delta_{0} \phi_{1}\left(\lambda_{1}\right)-\varepsilon_{1} \lambda_{1}^{2}=-\frac{159012}{125} \varepsilon-\left(A_{1}-\varepsilon_{1}\right) \varepsilon^{2}+O\left(\varepsilon^{3}\right),
$$




$$
\begin{array}{r}
\Delta_{0} \lambda_{2}=-\frac{159012}{125} \varepsilon-A_{2} \varepsilon^{2}+O\left(\varepsilon^{3}\right), \\
\Delta_{0} \phi_{0}\left(\lambda_{1}\right)=-\frac{159012}{125} \varepsilon+\frac{96935282}{3125} \varepsilon^{2}+O\left(\varepsilon^{3}\right),
\end{array}
$$

where $\phi_{1}\left(\lambda_{1}\right)$ and $\phi_{0}\left(\lambda_{1}\right)$ are defined in Theorem 2, and here $\lambda_{1}=\varepsilon$ and $\lambda_{2}=-(24 / 5) \varepsilon$. By some easy calculations, we can obtain that for $0<\varepsilon_{1}<A_{1}-A_{2}$ inequality $\Delta_{0} \phi_{1}\left(\lambda_{1}\right)-\varepsilon_{1} \lambda_{1}^{2}<$ $\Delta_{0} \lambda_{2}<\Delta_{0} \phi_{0}\left(\lambda_{1}\right)$ holds. Thus, by Theorem 2 we can get the result in this theorem.

\section{Conflict of Interests}

The authors declare that there is no conflict of interests regarding the publication of this paper.

\section{Acknowledgments}

The project was supported by National Natural Science Foundation of China (Grant no. 11271261), Grants from Ministry of Education of China nos. 20103127110001 and FP7PEOPLE-2012-IRSES-316338.

\section{References}

[1] J. Hofbauer and K. Sigmund, Ebolutionary Games and Population Dynamics, Cambridge Unibersity Press, Cambridge, UK, 1998.

[2] R. M. May, "Limit cycles in predator-prey communities," Science, vol. 177, no. 4052, pp. 900-902, 1972.

[3] R. M. May and W. J. Leonard, "Nonlinear aspects of competition between three species," SIAM Journal on Applied Mathematics, vol. 29, pp. 243-253, 1975.

[4] S. Smale, "On the differential equations of species in competition," Journal of Mathematical Biology, vol. 3, no. 1, pp. 5-7, 1976.

[5] D. Xiao and W. Li, "Limit cycles for the competitive three dimensional Lotka-Volterra system," Journal of Differential Equations, vol. 164, no. 1, pp. 1-15, 2000.

[6] E. C. Zeeman and M. L. Zeeman, "From local to global behavior in competitive Lotka-Volterra systems," Transactions of the American Mathematical Society, vol. 355, no. 2, pp. 713-734, 2003.

[7] J. Hofbauer and J. W.-H. So, "Multiple limit cycles for three dimensional Lotka-Volterra equations," Applied Mathematics Letters, vol. 7, no. 6, pp. 59-63, 1994.

[8] M. Gyllenberg, P. Yan, and Y. Wang, "A 3D competitive LotkaVolterra system with three limit cycles: a falsification of a conjecture by Hofbauer and so," Applied Mathematics Letters, vol. 19, no. 1, pp. 1-7, 2006.

[9] M. W. Hirsch, "Systems of differential equations that are competitive or cooperative. V. Convergence in 3-dimensional systems," Journal of Differential Equations, vol. 80, no. 1, pp. 94106, 1989.

[10] M. W. Hirsch, "Systems of differential equations which are competitive or cooperative IV: structual stability i three-dimensiona systems," SIAM Journal on Mathematical Analysis, vol. 21, pp. 1225-1234, 1990.
[11] M. L. Zeeman, "Hopf bifurcations in competitive threedimensional Lotka-Volterra systems," Dynamics and Stability of Systems, vol. 8, no. 3, pp. 189-216, 1993.

[12] Z. Lu and Y. Luo, "Three limit cycles for a tree-dimensional Lotka-Volterra competitive System with a heteroclinic cycle," Computers and Mathematics with Applications, vol. 46, no. 2-3, pp. 231-238, 2003.

[13] M. Bobieński and H. Żołądek, "The three-dimensional generalized Lotka-Volterra systems," Ergodic Theory and Dynamical Systems, vol. 25, no. 3, pp. 759-791, 2005.

[14] J. Llibre and D. Xiao, "Limit cycles bifurcating from a nonisolated zero-Hopf equilibrium of three-dimensional differential systems," to appear in Proceeding of American Mathematical Society.

[15] L. Dingjun, W. Xian, Z. Deming, and H. Maoan, Bifurcation Theory and Methods of Dynamical Systems, World Scientific, Singapore, 1997.

[16] M. Han, Periodic Solutions and Bifurcation Theory of Dynamical Systems, Science Press, Beijing, China, 2002. 


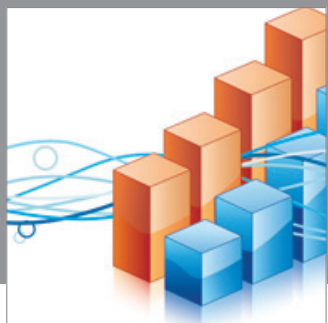

Advances in

Operations Research

mansans

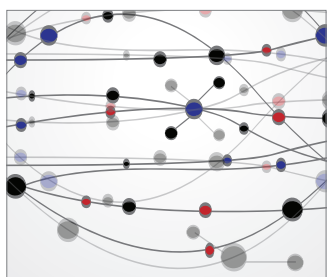

The Scientific World Journal
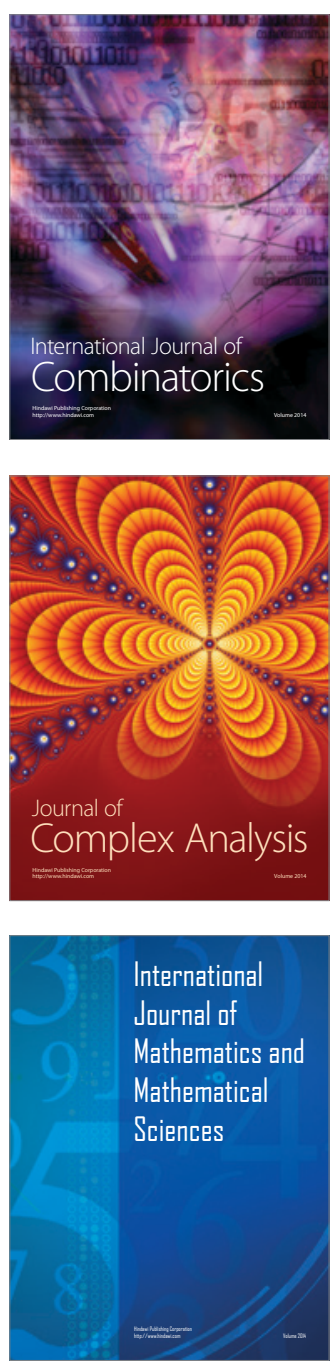
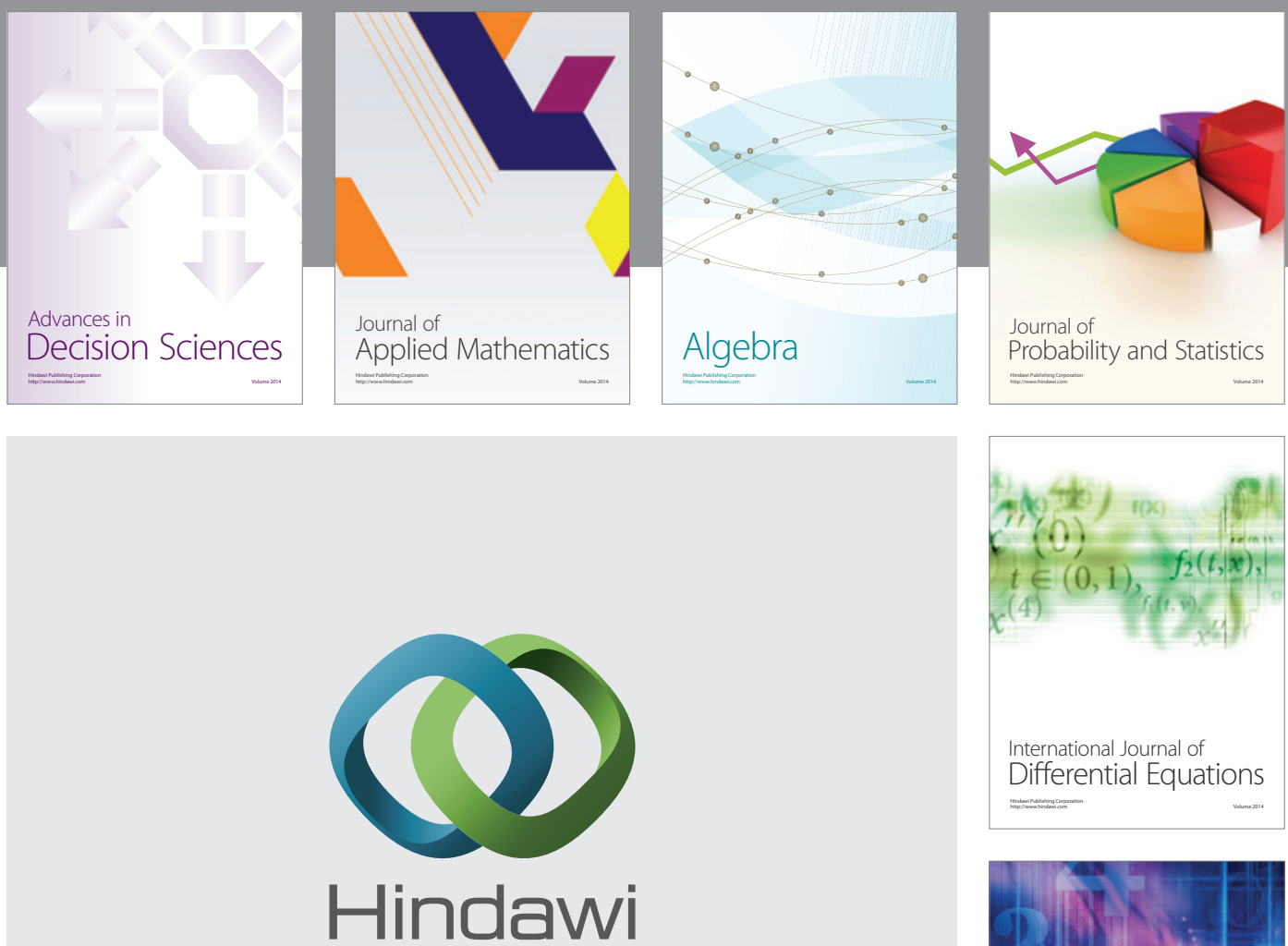

Submit your manuscripts at http://www.hindawi.com
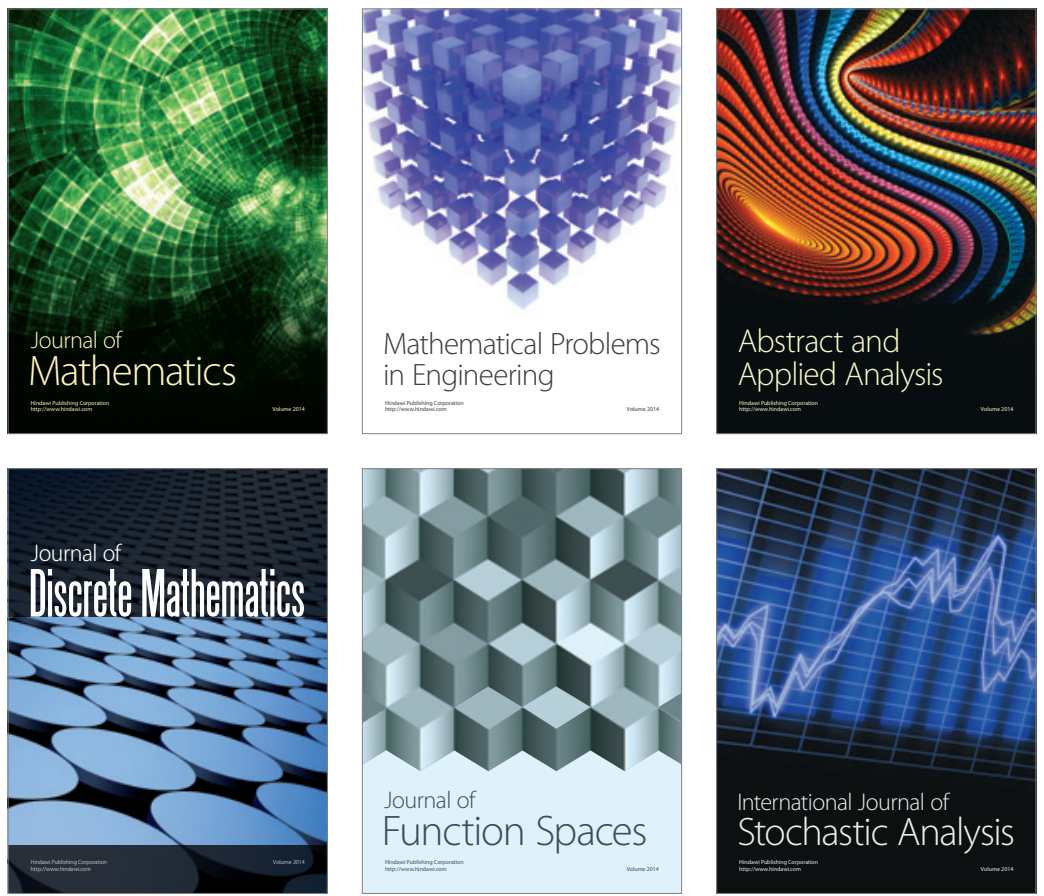

Journal of

Function Spaces

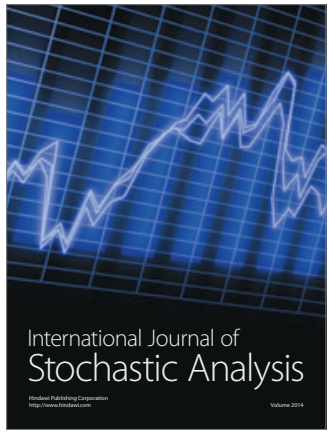

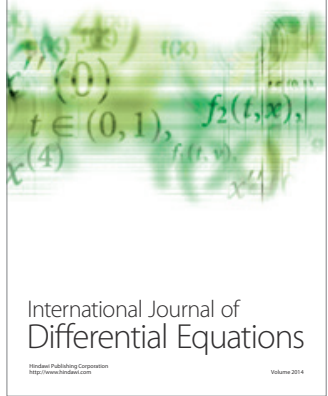
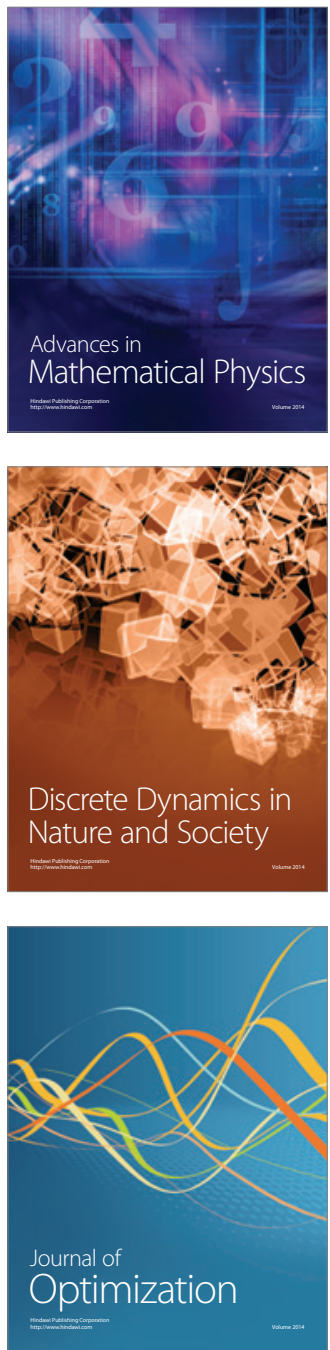